3 Research Square

\title{
A simultaneous feature selection and compositional association test for detecting sparse associations in high-dimensional metagenomic data
}

Andrew Hinton ( $\square$ andrew84@email.unc.edu )

University of North Carolina at Chapel Hill School of Medicine https://orcid.org/0000-0001-6878-2289

Peter J. Mucha

Dartmouth College

\section{Research Article}

Keywords: Microbiome Association Study, Sparse Association Signals, Pairwise Log Ratios, Compositional Data, Multivariate Analysis, Feature Selection, Network Analysis

Posted Date: July 12th, 2021

DOl: https://doi.org/10.21203/rs.3.rs-703177/v1

License: (c) (1) This work is licensed under a Creative Commons Attribution 4.0 International License.

Read Full License 
1 A simultaneous feature selection and compositional association test for detecting sparse

2 associations in high-dimensional metagenomic data

3 Andrew L. Hinton ${ }^{1,2, *}$ and Peter J. Mucha ${ }^{1,3,4}$

$4 \quad *$ Correspondence: andrew84@email.unc.edu

$5{ }^{1}$ Curriculum in Bioinformatics and Computational Biology, University of North Carolina, Chapel Hill, NC, 6 USA

$7{ }^{2}$ University of North Carolina at Chapel Hill Food Allergy Initiative, School of Medicine, Chapel Hill, NC,

8 USA

$9{ }^{3}$ Departments of Mathematics and Applied Physical Sciences, University of North Carolina, Chapel Hill, 10 NC, USA

$11{ }^{4}$ Department of Mathematics, Dartmouth College, Hanover, NH, USA

12 


\section{Abstract (350 words)}

22 Background: Numerous metagenomic studies aim to discover associations between the microbial

23 composition of an environment (e.g. Gut, Skin, Oral) and a phenotype of interest. Multivariate analysis

24 (MVA) is often performed in these studies without critical a priori knowledge of which taxa are associated with the phenotype being studied. Consequently, non-parametric MVA methods are applied directly to all taxa surveyed independent of noise. This approach typically reduces statistical power in

27 settings where true associations among only a few taxa are obscured by high dimensionality (i.e. sparse association signals). At the same time, the inclusion of all taxa can confound the extraction of key biological insights. Further, low sample size and compositional sample space constraints exist in these data whereby beyond-study generalizability may be reduced if not properly accounted for. More powerful association tests that are interpretable and directly account for compositional constraints while detecting sparse association signals are needed.

Methods: We developed Selection-Energy-Permutation (SelEnergyPerm), a non-parametric group

34 association test with embedded feature selection. SelEnergyPerm directly accounts for compositional 35 constraints by selecting parsimonious log ratio signatures from the set of all pairwise log ratios (PLR)

36 between features (OTUs, taxa, etc.). To do this, network methods are used to rank, select, and maximize

37 the between-group association of a candidate log ratio subset. This process is then repeated with an appropriate permutation testing design to simultaneously determine the significance of the selected

39 signatures and association.

Results: Simulation results show SelEnergyPerm selects small independent sets of log ratios that capture

41 strong associations in a range of scenarios with small and large dimensional feature spaces. Additionally, 42 our simulation results demonstrate SelEnergyPerm consistently detects/rejects associations in synthetic 
data with sparse, dense, or no association signals. We demonstrate the novel benefits of our method in

44 four case studies utilizing publicly available $16 \mathrm{~S}$ rRNA and whole-genome sequencing datasets.

45 Conclusions: Tools to analyze complex high-dimensional metagenomic datasets with sparse association

46 signals using robust PLR have not been sufficiently developed previously. We propose SelEnergyPerm, a

47 novel framework for the discovery of phenotype-associated, metagenomic log ratio signatures for

48 characterizing and understanding alterations in microbial community structure. SelEnergyPerm is

49 implemented in R, available at https://github.com/andrew84830813/selEnergyPermR.

50 Keywords: Microbiome Association Study, Sparse Association Signals, Pairwise Log Ratios,

51 Compositional Data, Multivariate Analysis, Feature Selection, Network Analysis

\section{Background}

53 Metagenomic studies have enabled unprecedented insight into connections between microbes, their

54 functions, and human disease [1]. These insights are a direct result of rapid advances in next-generation

55 sequencing technologies which are critical to metagenomic studies. Specifically, these technologies are

56 leveraged in two popular approaches: $16 \mathrm{~S}$ ribosomal rRNA amplicon (16S) and whole-genome shotgun

57 (WGS) sequencing [2]. Application of these approaches are widespread and have been used to study

58 associations between the gut microbiome composition and Colorectal Cancer, IBD, Obesity, Cirrhosis,

59 and anxiety/depression in humans via the gut-brain axis to name a few [3-7]. The skin, oral, and nasal

60 microbiomes among other sites have also been studied in connection to disease onset and progression

61 [8-10]. With an increasing number of putative associations between microbial communities from various

62 sites of the human body and disease being reported, microbial compositions are now being explored as

63 diagnostic and screening tools $[11,12]$. While exciting, appropriate statistical methods are still needed

64 to overcome methodological challenges in these exceptional data, so that robust microbial biomarkers 
and true associations can be discovered among noisy high-dimensional metagenomic data where sample sizes in observational studies are smaller than the number of features discovered.

67 Before metagenomic data can be used to test for associations, raw sequencing data must be appropriately processed. Taxonomic count tables are created by processing raw $16 \mathrm{~S}$ or WGS sequencing data through bioinformatics pipelines such as QIIME [13] or mothur [14] for amplicon sequencing data and MetaPhIAn2 [15] or Kraken [16] for WGS data. Sequencing reads are assigned a taxonomy where

71 the resulting count tables are then used to profile and analyze the association between groups under 72 study at various taxonomic levels (Phylum - Species). These data are often sparse and summarize the 73 total number of reads for each taxonomic assignment within each sample. In current practice, total 74 counts in these settings have been widely recognized as being uninformative due to limitations within 75 sequencing technology [17-19]. These data are considered to carry only relative information whereby 76 special statistical techniques and considerations are required. That is, these relative data have a unit-

77 sum simplex sample space where traditional Euclidean-based statistical methods have limited

78 applicability due to geometrical differences between sample spaces. Ignoring these constraints has been 79 shown to increase type I error [19], increase the chance of reporting spurious [20] associations, and thus 80 may limit the ability of biomarkers (discovered from relative abundance) to generalize beyond studies.

81 A direct way to address simplex sample space constraints imposed by relative data is through a log-ratio

82 transformation. This transformation emerged from the statistical analysis of compositional data [21] and

83 functions by mapping relative data from the unit-sum simplex to traditional Euclidean space.

84 Importantly, the log-ratio transformation is sub-compositionally coherent [21, 22], independent of the 85 number of dimensions (taxa, OTU's, etc.) observed in a cohort whereby true associations in the log-ratio 86 form are preserved. This is not true for relative abundance where proportions change as new 87 dimensions (OTU, Taxa, etc.) are considered, discovered, or removed. Further, sub-compositional 88 coherence is of practical importance in biomedical studies where biomarker discovery, disease 
prediction, and beyond-study generalization are paramount. While the log-ratio transformation is well-

90 known and routinely applied in some fields [23], its use in metagenomic datasets has been limited

91 despite its advantages. Indeed, significant barriers exist when applying a log-ratio transformation to

92 metagenomic data. Core challenges include properly handling zeroes [24, 25], selection and

93 interpretation of various log-ratio forms [21, 26-27], and scale differences in counts [28].

94 While the importance of the compositional nature of metagenomic data has recently been recognized

$95[17,29]$, few multivariate statistical methods have been developed directly for these data. Currently,

96 several univariate methods (ALDEx2, ANCOM, ANCOM-BC) for detecting differential abundance in

97 compositional metagenomic data have been proposed [30-32]. While powerful, these methods are

unable to detect multivariate structure within complex interconnected microbial communities [33, 34].

99 Complicated microbial patterns, which occur in settings where there are significantly more variables

100 than samples, can however be discovered with appropriate network and multivariate statistical

101 methods. Further, multivariate statistical methods, which are appropriate when there exist relationships

102 between a set of variables (i.e. microbial composition) and two or more groups are to be analyzed, have

103 better control over type I error [34].

104 Currently, several multivariate statistical methods to detect between-group distributional differences or

105 associations in metagenomic data can be used. A subset of these methods are universal and require a

106 suitable beta diversity or between-sample distance (Euclidean, Manhattan, Mahalanobis, etc.) or

107 dissimilarity (Bray-Curtis, weighted/unweighted UNIFRAC, Jaccard, etc.) metric be specified before

108 analysis. PERMANOVA [35], ANOSIM [36], and/or the Energy distance [37], which are nonparametric

109 tests, can then be applied to test distributional differences between groups. Between-group association

110 signals in metagenomic data may be sparse, i.e., result from differences among only a few features

111 (OTU, taxa, etc.) or dense where the association signal is formed by differences between many features. 
112 Importantly, the above-mentioned nonparametric tests lack embedded feature selection and thus can

113 have limited statistical power for detecting sparse signals in high-dimensional data.

114 Feature selection, which is essential to detecting sparse association signals in sparse high-dimensional

115 metagenomic data [38,39], requires sophisticated methods and care to simultaneously select features

116 and test associations while maintaining reasonable type I error control $[40,41]$. To address this need,

117 the adaptive microbiome-based sum of powered score (aMiSPU) method was proposed to test

118 between-group associations with embedded feature selection through the variable ranking of

119 untransformed relative abundances [38]. However, aMiSPU does not inherently account for the

120 compositional structure of the data and requires OTU's and phylogenetic tree to test associations which

121 may not always be available (e.g. WGS data). Further, aMiSPU lacks automatic feature selection across

122 all tests and was shown to be underpowered when applied to highly sparse data [39]. Similarly, OTU's

123 and phylogenetic information are also required as input for microbiome higher criticism analysis (MiHC)

124 [39], which like aMiSPU tests sparse associations in 16S data using untransformed relative abundances

125 and lacks automatics feature selection. Moreover, MiHC results report an arbitrary top number of OUT's

126 to be important thus making it difficult to understand which features specifically contribute to the

127 overall detected association.

128 Inspired from concepts put forth in the Direction-Projection-Permutation method for assessing

129 statistical significances in high-dimensional settings [43], we introduce here the Selection-Energy-

130 Permutation (SelEnergyPerm) method for testing and understanding associations in sparse 16S and WGS

131 data. SelEnergyPerm is the first method to our knowledge to utilize robust pairwise log ratios to detect

132 and understand parsimonious log ratio signatures from all types of metagenomic data through

133 simultaneous feature selection and association testing. We first show that our novel approach selects

134 smaller subsets of non-redundant log ratios that better maximize between-group associations when

135 compared to popular feature selection methods. Next, we show through an extensive simulation study 
136 (using synthetic and empirical 16S/WGS data distributions) that SelEnergyPerm has, on average, better

137 combined power and false discovery control via the Matthews Correlation Coefficient (MCC) when

138 compared to existing beta-diversity-based approaches. Finally, to demonstrate the utility of

139 SelEnergyPerm in detecting and understanding differences between metagenomic distributions, we

140 apply our method in four case studies utilizing publicly available metagenomic datasets where we test

141 associations between: (1) Ugandan infant's cerebrospinal fluid microbiomes and post-infectious

142 hydrocephalus, (2) delivery mode and the composition of infant's gut microbiomes over the first three

143 months of life, (3) adult gut microbiomes and abnormal fecal calprotectin levels, and (4) the gut

144 microbiome composition of infants within the first 6 months of life and future food allergy to egg, milk,

145 or peanuts.

\section{Results}

147 The Selection-Energy-Permutation framework for simultaneous feature selection and group 148 association testing.

149 To robustly uncover sparse microbial signatures while simultaneously testing multivariate group

150 associations, we based our SelEnergyPerm framework on a novel network-based feature selection

151 approach combined with permutation testing for sparse high-dimensional low-sample-size

152 compositional metagenomic data. Our framework (Figure 1A), which selects from all pairwise log ratios

153 between variables (Taxa, OTU's, etc.), first scores the between-group variation of individual log ratios

154 using our Differential Compositional Variation (DCV) scoring measure (see Methods). From this, a

155 weighted DCV log-ratio network is formed and subsequently pruned to reduce redundancy and

156 complexity via a maximum spanning tree. Final subsets are then selected by maximizing the between-

157 group association using a greedy forward stepwise selection procedure. Multivariate test statistics,

158 which measure the strength of the association between groups, are then computed on the final retained 
subset. Statistical significance is determined by repeating this process with permuted group labels to

160 obtain the permutation distribution of the test statistic of interest under feature selection. In this way,

161 we determine if the observed association is larger than what would be expected by chance (Figure 1B).

162 To this end, our framework tests the overall null hypothesis of no association between the metagenomic

163 composition and group labels.

\section{Feature selection comparison to other methods.}

165 We first benchmarked the multivariate characteristics of subsets selected by our feature selection (FS)

166 approach against a set of popular methods for FS: Boruta [43], Least Absolute Shrinkage and Selection

167 Operator (LASSO) [44], Information Gain Filtering [45], and Random Forest Recursive Feature

168 Elimination (RFE) [46]. The benchmarks were carried out by varying the number of log-ratio dimensions

169 in the full feature set using five simulation scenarios with both balanced and unbalanced sampling

170 designs (see Methods). Specifically, for subsets returned by each method, we studied the number of log

171 ratios selected (as a proxy for model complexity), the clustering coefficient of the log-ratio network

172 (measuring log-ratio redundancy), and the combined F-statistic (strength of association, see Methods),

173 and the computational time required to return the final subset (Additional file 1: Figure S1). In Figure 2,

174 we present results from scenarios with a balanced sampling design. Notably, the results for the

175 unbalanced sampling design scenarios are similar and do not change the overall comparative

176 interpretation (Additional file 2: Figure S2). Examination of the clustering coefficient across all simulation

177 scenarios/dimensions demonstrates that SelEnergyPerm consistently selects linearly independent

178 subsets of log ratios (Figure 2 and Additional file 2: Figure S2, clustering coefficient $=0$ ), in contrast with

179 the subsets observed in other methods tested. Of note, a clustering coefficient $>0$ indicates a selected

180 log-ratio subset contains at least one triple of linearly-dependent log ratios (closing a triangle in the log-

181 ratio network), thereby unnecessarily increasing dimensionality and model complexity. (We note that

182 any cycle present in a log-ratio network indicates linear dependence, though we did not test for cycles 
larger than closed triangles, and in particular we emphasize that by construction the SelEnergyPerm-

184 selected subsets do not include any such cycles.) Additionally, the number of log ratios retained by each method across every scenario tested revealed subsets selected by SelEnergyPerm were, on average, 14 to 149 times smaller than other methods (Figure 2 and Additional file 2: Figure S2).

187 Next, the strength of the association, measured by the combined-F statistic (see Methods), indicates SelEnergyPerm-selected subsets typically capture higher between-group variations than other methods tested. In particular, in Scenarios 2-4, SelEnergyPerm subsets were observed to have, on average,

190 higher combined- $\mathrm{F}$ values than all other methods across all dimensions tested (Figure 2 and Additional 191 file 2: Figure S2). Meanwhile, in Scenarios 1 and 5, SelEnergyPerm subsets generally performed similarly 192 to the other methods but better as the dimensionality increased. Notably, Scenarios 1 and 5 do not 193 simulate sparse association signals and have strong between-group dispersion effects present. These 194 results indicate SelEnergyPerm returned subsets better capturing sparse associations (Scenarios 2-4) 195 than the other FS methods tested. Computational time experiments show, across all scenarios tested, 196 SelEnergyPerm is on average faster than Boruta and RFE but slower than LASSO and Information Gain

197 filtering (Additional file 1: Figure S1). Overall, SelEnergyPerm subsets were non-redundant, significantly 198 more parsimonious, and captured stronger associations than other methods tested, thereby enabling 199 robust biological interpretation using log ratios in high-dimensional feature spaces.

\section{Detection of sparse associations in synthetic data.}

201 Here, we use data simulated from theoretical distributions to compare the ability of SelEnergyPerm, 202 PERMANOVA, ANOSIM, and the energy test to detect associations in sparse high-dimensional data. That 203 is, we are interested in determining how well each method accepts or rejects the null hypotheses (no 204 difference between groups) when presented with two groups of data that, as ground truth, come from 205 the same (Null Case; Type I error assessment) or different (True Case; power assessment) distributions. 
From this, we report the performance of each method in terms of the Matthews correlation coefficient

207 at $\alpha=0.05$ (see Methods) for 4 simulation scenarios (see Methods) with both balanced and unbalanced

208 sampling designs (Figure 3). For brevity, we shall refer to the collection of PERMANOVA, ANOSIM, and

209 energy tests as the standard methods.

210 In Scenario 1, where data are simulated from a Dirichlet distribution with between-group location and

211 dispersion effects that grow as the number of dimensions increase (see Methods), we see for the

212 balanced design that both SelEnergyPerm and the energy test perform well over all dimensions (number

213 of log ratios) tested. Notably, ANOSIM loses the ability to detect associations as the number of

214 dimensions increases while PERMANOVA performs poorly over all dimensions. The poor performance of

215 ANOSIM and PERMANOVA is directly attributable to the underlying heterogeneity of variance present in

216 the data generated in this scenario. These limitations of PERMANOVA and ANOSIM have also been

217 discussed [47]. The presence of dispersion effects is confirmed with the PERMDISP2 [48] method and

218 can be observed to be steady (Figure 3 - Scenario 1) and increasing across dimensions. For the

219 unbalanced design, SelEnergyPerm and the energy test both retain strong performance and have

220 comparable performance over most dimensions, whereas ANOSIM completely loses the ability to detect

221 associations under the unbalanced design and PERMANOVA continues to perform poorly across all

222 dimensions

223 For Scenario 2 (Figure 3), the data distributions for each group are simulated from two Dirichlet

224 distributions that differ in the location of the first component and overall variance. That is, this scenario

225 embeds a sparse signal (location shift) in the first dimension with random noise in the remaining

226 dimensions. The results for this scenario show that for the balanced case SelEnergyPerm performs

227 significantly better than all other methods tested. For the unbalanced case, SelEnergyPerm performs

228 better than all other methods for smaller numbers of dimensions, however, it performs similarly to 
ANOSIM as the number of dimensions increases. Notably, the performance of ANOSIM improves as the

230 number of dimensions increases for both the balanced and unbalanced cases.

231 For Scenario 3 (Figure 3), the data distributions for the first class are simulated from the additive logistic

232 normal distribution. Data for the second class are also generated from an additive logistic normal

233 distribution with the same parameters (same covariance matrix) but with location shifts in the first $25 \%$

234 of the dimension. Under this scenario, we observed the performance of SelEnergyPerm to be

235 comparable to the standard methods for the balanced case and slightly worse than the standard

236 methods for the unbalanced case. The reduced performance in the unbalanced case is attributable to

237 the dense signal ( $25 \%$ of feature) being in direct tension with the reduced feature selection subset

238 objective of SelEnergyPerm.

239 Lastly, in Scenario 4 (Figure 3), a location shift only (same between class covariance structure) was

240 embedded in the first component of two additive logistic normal distributions that increases with the

241 number dimensions. Here, SelEnergyPerm outperformed the standard methods as the number of

242 dimensions increased for both the balanced and unbalanced cases. While performing better overall

243 relative to the standard methods, a notable decrease in performance was observed from the balanced

244 to the unbalanced case for SelEnergyPerm. This decrease in performance was exacerbated among the

245 standard methods where performance not only decreased between sampling designs but also generally

246 declined as the number of dimensions increased in the unbalanced design.

247 Overall sparse association detection performance as measured by MCC, sensitivity, specificity, positive

248 predictive value, negative predictive value, Youden index, and false-positive rate across all scenarios at

249 an $\alpha=0.05$ are shown in Additional File 3: Figure S3. These aggregate results demonstrate

250 SelEnergyPerm in general outperforms the standard methods for detecting sparse associations under

251 the synthetic data simulation scenarios presented here. 
253 To further assess performance, we benchmarked our method against the standard methods on data

254 simulated from properties observed in real metagenomic datasets. In this way, unique metagenomic

255 data characteristics such as sparsity, over dispersion, and complex co-occurrence patterns are assessed

256 synthetically. As described in the detection of sparse associations in the synthetic data section above,

257 MCC is used to assess the ability of each method to detect associations across these settings.

258 In the first setting, (Figure 4-16S/WGS: Increasing Covariance Diff.), an increasing covariance effect 259 with a decreasing location effect between classes was simulated using healthy subsets of 16S and WGS 260 samples. The increasing dispersion effect is confirmed with PERMDISP2 for both sampling designs

261 (Figure 4). For 16S and WGS data with a balanced sampling design, SelEnergyPerm outperforms the 262 standard methods across all effect sizes and has strong performance as the number of dimensions 263 increase. For $16 \mathrm{~S}$ data with an unbalanced design, all methods performed poorly as the location shift 264 effect increases. This trend is traceable to the strong embedded covariance effect between classes 265 which is a known confounder in high-dimensional association settings [47]. Notably, only SelEnergyPerm 266 and ANOSIM maintain positive MCCs on average, indicating these methods better control type I error 267 (with severely limited power) under this sampling design. For WGS data with an unbalanced design, 268 SelEnergyPerm outperformed the standard methods and had better association detection across all 269 effect levels.

270 For the second simulation setting, (Figure 4-16S/WGS: Increasing Location Effects), we simulated large 271 location shifts between classes by increasing the size of the association signal from $5 \%$ to $50 \%$ of all

272 features with fixed covariance structures. These shifts were computed using synthetic subsets of WGS

273 and 165 samples from publicly available healthy gut microbiomes. Indeed, PERMDISP2 analysis

274 confirmed the absence of covariance effects. For both 165 and WGS data with a balanced sampling 
275 design, SelEnergyPerm outperformed all standard methods. As expected, in both WGS and 16S data, the

276 performance of the standard methods increased as the association signal became less sparse. Again, for

277 the unbalanced design in both WGS and 16S data, SelEnergyPerm outperformed all standard methods.

278 Importantly, the detection ability of the standard methods improved as the association signal became

279 less sparse.

280 Finally, overall sparse association detection performance metrics are shown in Additional File 4: Figure

281 S4. These aggregate results demonstrate SelEnergyPerm has better overall sparse association detection

282 performance when compared to standard methods using data simulated from real 16S and WGS

283 datasets.

284 Microbial association between Ugandan infant's cerebrospinal fluid microbiomes and post-infectious 285 hydrocephalus.

286 In this case-control study by Paulson et al., the cerebral spinal fluid (CSF) of Ugandan infants was

287 profiled using $16 \mathrm{~S}$ rRNA amplicon sequencing to characterize microbial agents associated with Post

288 Infectious Hydrocephalus (PIH) following neonatal sepsis [49]. This processed gut microbiome dataset

289 was retrieved from microbiomeDB $[50,51]$ and consisted of 369 distinct taxa measured on 92 samples

290 (58 PIH and 34 Non-Post Infectious Hydrocephalus (NPIH) patients). Removing taxa not present in at

291 least $10 \%$ of samples yielded 57 total distinct taxa (i.e., 1,596 taxa log ratios). We then apply

292 SelEnergyPerm to determine if there was an association between the microbiome composition in the

293 CSF and PIH/NPIH disease status. Further, we utilize the reduced SelEnergyPerm log-ratio signature of

294 PIH in CSF to gain insight into specific microbiome compositional differences.

295 From this, we confirm with SelEnergyPerm a significant association ( $\mathrm{cF}=33.59817$, empirical $p=0.007$ )

296 exists between the composition of microbes in the CSF and PIH/NPIH [49] (Figure 5A). Our method

297 identified a reduced log-ratio signature of 12 ratios between 13 taxa as being significantly associated 
with PIH/NPIH (Figure 5B). Random forest (RF) models were used to understand the capability of the

SelEnergyPerm signature for discriminating between disease statuses. Indeed, estimating the discriminatory ability with RF using 50 repeats of 10 -fold cross-validation, we computed an Area Under the Receiver Operating Characteristic Curve (AUC) $=0.906$ (0.879-0.935 95\% Cl) (Figure 5C). We emphasize, however, that the more complex RF models with all 1,596 pairwise log ratios yielded a comparable AUC = $0.892(0.860-0.92395 \% \mathrm{Cl}$ ) (Figure 5C). In addition, microbiome analysis carried out

304 in Paulson et al. revealed Paenibacillus alone to be important for predicting PIH. Here, using only the 305 relative abundance of Paenibacillus with RF, we observed an AUC $=0.830(0.792-0.86795 \% \mathrm{Cl})$ which 306 was significantly lower than the SelEnergyPerm results. Combined, these results suggest the 307 parsimonious SelEnergyPerm-derived log-ratio signature retains important disease interactions and 308 better discriminates between PIH vs. NPIH when compared to Paenibacillus alone.

309 To understand how the log ratios in our signature work together to explain differences between the CSF 310 microbiome of PIH vs. NPIH patients we apply principal component analysis (PCA) (Figure 5D) and 311 analyzed the means of the log ratios. From that, examination of the distribution of samples shows the 312 greatest separation between disease groups occurs along PC1 (Figure 5D) which explains $78.48 \%$ of the 313 total variation. This separation indicates positive scores along PC1 are associated with non-PIH whereas 314 negative scores are associated with PIH samples. Further, analyses of the log ratio mean between 315 groups for each log ratio in the SelEnergyPerm signature indicate the abundance of Paenibacillus is 316 significantly increased (Figure 5E) relative to taxa it's connected to (Figure 5B). Moreover, RF variable 317 importance indicates the log ratio between Paenibacillus relative to Pseudomonas to be most important 318 for distinguishing between disease statuses. Indeed, analysis of PC1 loadings (Figure 5E) reveals 319 increased abundance of Pseudomonas relative to Paenibacillus results in positive loadings (non-PIH 320 associated) along PC1. Overall our results confirm, using pairwise log ratios derived from SelEnergyPerm, 321 the importance of Paenibacillus in PIH. Additionally, we show the interaction between the abundance of 
322 Pseudomonas relative to Paenibacillus is particularly important whereby more Pseudomonas is

323 characteristic of non-PIH and more Paenibacillus is associated with PIH.

324 Association between delivery mode and the composition of infant's gut microbiomes over the first 325 three months of life.

326 Bokulich et al. monthly profiled the gut microbiome of infants with either a vaginal or cesarean delivery 327 mode using 16S rRNA sequencing for the first two years of life [52]. The processed dataset was retrieved 328 from the QIITA repository using study ID 10249 [53]. Specifically, we extracted samples during the first 3 329 months of life which totaled 230 samples from 63 infants (Cesarean $=25$, Vaginal $=38$ ). We aggregated 330 OTU's to the family-genus level which resulted in 140 distinct taxa (9,730 log ratios) present in at least $33110 \%$ of all samples by month. Here we apply SelEnergyPerm to determine if the gut microbiomes are 332 different between the delivery modes of infants at any of the first 4 monthly time points collected (0-3 333 months). Secondarily, we studied our reduced log ratio signatures to understand gut microbiome 334 compositional differences between delivery modes at time points where significant differences were 335 detected.

336 Applying SelEnergyPerm to each time point with restricted permutation testing to account for repeated 337 host microbiomes within a collection month and correcting for multiple comparisons using the 338 Benjamini-Hochberg $(\mathrm{BH})$ procedure, we found significant differences in the composition of the gut 339 microbiomes between delivery modes during the 0-2 month collection periods (Figure 6A). Notably, 340 restricted permutation testing with PERMANOVA and ANOSIM using all taxa PLR failed to detect 341 differences between the gut microbiomes at $\alpha=0.05$. This is also clear when comparing the 342 discriminatory ability of each set. Using Partial Least Squares Discriminate Analysis (PLS-DA) with 343 repeated cross-validation stratified by both delivery mode and host, we observed the AUC of the 344 SelEnergyPerm-derived signatures to be higher across all time points when compared to models trained 
using all PLR (Figure 6B). We next used the reduced log-ratio signatures and their PLS-DA variable

importance scores to better understand which taxa are most important for discriminating between

347 delivery modes. Indeed, aggregating to the family level for ease of interpretation, we found during

348 months 0 and 1 that Bacteroidaceae were top contributors to compositional differences (Figure 6C). This

349 pattern changed during month 2 where Rikenellaceae taxa were most important for discriminating

350 between delivery modes (Figure $6 \mathrm{C}$ ). Finally, to understand the direction of these differences (i.e. is the

351 numerator more abundant than denominator or vice-versa between groups for a given log ratio), we

352 examine the log-ratio means networks using the SelEnergyPerm signature. Here, we analyze the

353 directed log ratio means network relatively (i.e. taxa A more/less abundant than taxa B) between

354 delivery modes (Figure 6D). Specifically, given the hub-spoke character, with a single highly connected

355 and central node in the directed MST formed by the SelEnergyPerm signature, we can see month 0 is

356 dominated by differences between log ratios that include Lachnospira, and Bacteroides. In, particular

357 Lachnospira and Bacteroides are more abundant relative to their network of taxa connections for infants

358 with a vaginal delivery mode whereas the opposite is true for infants with a cesarean delivery mode. For

359 month 1, Bacteroides are observed to be more abundant relative to its network of taxa connections for

360 infants with a vaginal delivery mode. The opposite is true for infants with a Cesarean delivery mode

361 where Bacteroides are less abundant within its network of taxa connections. Finally, for month 2,

362 Rikenellaceae taxa can be observed to be more abundant than both Clostradiacea and Proteus taxa

363 where the opposite is true for infants with a Cesarean delivery mode.

364 Association between abnormal fecal calprotectin levels and the composition of the gut microbiome in

365 healthy and IBD individuals.

366 Here we apply SelEnergyPerm to analyze WGS microbiome data from the integrative human

367 microbiome project (iHMP) [54], a longitudinal study designed to uncover interactions between disease

368 and human-associated microbial communities. Specifically, using the inflammatory bowel disease (IBD) 
part of the iHMP study, we tested whether there exists an association between the gut microbiome

370 composition and abnormal levels of fecal calprotectin, a protein marker of intestinal inflammation [54].

371 Processed microbiome data were extracted from the Inflammatory Bowel Disease Multiomics Database

372 [55] resulting in 399 samples (93 individuals) reporting fecal calprotectin levels that were above 120

373 (abnormal; $n=190$ ) or below 50 (normal; $n=209)$. There were 122 species identified $(7,381$ log ratios)

374 as being present in at least $10 \%$ of all samples.

375 Using restricted permutation testing, accounting for the order of visit and diagnosis of Ulcerative Colitis

376 (UC), Crohn's Disease (CD), or non-IBD, SelEnergyPerm identified a significant association (cF $=92.507, p$

$377=0.000999,1000$ permutations) between the composition of the gut microbiome and abnormal levels

378 of fecal calprotectin in corresponding stool samples (Figure 7A). Notably, both ANOSIM and

379 PERMANOVA with restricted permutation designs using all log ratios detected this association. To

380 assess whether the associated SelEnergyPerm log-ratio signature (31 log ratios between 26 species)

381 retained enough information to adequately discriminate between levels of fecal calprotectin we

382 estimated the discriminatory ability both using the reduced signature and using all log ratios. Using

383 repeated cross-validation with PLS-DA we found the SelEnergyPerm signature (AUC $=0.829$ (0.803-

$3840.85495 \% \mathrm{Cl})$ ) to have comparable performance to PLS-DA models trained using all log ratios (AUC $=$

$3850.833(0.803-0.86295 \% \mathrm{CI}))($ Figure 7B). Examination of the latent space projection of a final PLS-DA

386 model fit using the SelEnergyPerm signature reveals strong separation between individuals with normal

387 vs. abnormal fecal calprotectin levels (Figure 7C). A directed log-ratio network of the SelEnergyPerm

388 signature weighted by PLS-DA variable importance shows log ratios involving Dialister invisus,

389 Streptococcus salivarius, Bacteroides fragilis, Escherichia coli, and Blautia wexlerae to be most important

390 for discriminating between levels of fecal calprotectin (Figure 7D). Interestingly, stratifying the log-ratio

391 signature by diagnosis reveals both shared (significant between diagnosis differences across all groups)

392 and distinct (significant between diagnosis differences among a single group) gut microbiome 
differences (Figure 7E). Particularly increased abundance of Dialister invisus relative to Bacteroides

394 ovatus, Intestinimonas butyriciproducens, and Anaerotignum lactatifermentans was observed to be associated with abnormal fecal calprotectin independent of diagnosis.

Association between the gut microbiomes of infants in early life and the development of allergenspecific sensitization

In this case study, we apply SelEnergyPerm to WGS gut microbiome data from the DIABIMMUNE study

[56]. The focus of this longitudinal study was to characterize interactions between the immune system and the gut microbiome in the context of autoimmunity and allergy. Specifically, the gut microbiomes of 401 infants from Finland, Russia, and Estonia were profiled monthly during the first 3 years of life. Processed 402 WGS data were directly accessed from [57]. Here we apply SelEnergyPerm to test if associations exist between the composition of the gut microbiome at 6-month intervals during the first 2 years of life and

404 allergy status. Allergy status was defined as food allergy (FA) if the host reported an allergy to egg, 405 peanuts, and/or milk at year 2 or non-FA otherwise [56]. We extracted 646 samples from 192 infants 406 (Russia $=53$, Finland $=70$, Estonia $=59)$ across 170 unique species $(14,365$ log ratios $)$.

407 Using restricted permutation testing to account for repeated host microbiomes and host country we 408 applied SelEnergyPerm to each timeframe and corrected for multiple comparisons using the BH procedure. We found significant differences in the composition of the gut microbiomes between allergy

410 status during both the first 6 months and the 6 - 12 month collection periods (Figure 8A). PERMANOVA 411 and ANOSIM using all taxa PLR detected differences between the gut microbiome during the first 6

412 months of life but did not detect differences between the gut microbiomes during the remaining 413 timeframes at an $\alpha=0.05$ after correcting for multiple comparisons with the $\mathrm{BH}$ procedure. This is 414 apparent when comparing the discriminatory ability between the SelEnergyPerm signature and all log 415 ratios. Using Partial Least Squares Discriminate Analysis (PLS-DA) with repeated cross-validation 
stratified by allergy status, host, and month, we observed the AUC of the SelEnergyPerm-derived

417 signatures to be significantly higher across all time points when compared to models trained with all log

418 ratios (Figure 8B). Using the SelEnergyPerm log ratio signatures and the corresponding PLS-DA variable

419 important scores we next examine which taxa are important for discriminating between food allergy

420 statuses later in life. Stratifying by month and selecting the top 5 strength (weighted degree) species

421 from our variable importance log-ratio network, we found during the first 6 months of life Clostridium

422 ramosum, Streptococcus parasanguinis, and Bifidobacterium bifidum to be major contributors of DCV

423 between allergy status (Figure $8 \mathrm{C}$ ). However, for the 6-12 month time period we found the abundance

424 of Clostridium hathewayi, Bacteroides dorei, and Haemophilus haemolyticus to be major contributors of

425 DCV (Figure 8C). A review of the log-ratio mean networks (Figure 8D) between allergy status during the

426 first 6 months shows Clostridium ramosum is, in general, more abundant relative to species (node

427 strength indicated by size) it is connected to in infants with FA vs. non-FA. Further, during the $6-12$

428 month time period we see more distinct differences in the log-ratio mean networks whereby

429 Bacteroides dorei can be observed to be more abundant relative to species it is connected to in FA

430 infants. We also observe Clostridium hathewayi to be more abundant than the species it is connected to

431 in infants with FA where the opposite is true in infants without FA.

\section{Methods and materials}

433 Selection-Energy-Permutation (SelEnergyPerm) for simultaneous feature selection and group

434 association testing in sparse high-dimensional compositional data.

435 In this section, we explain the SelEnergyPerm framework in greater detail. First, we describe our all

436 pairwise log ratios DCV scoring measure and then detail the construction of the weighted DCV networks.

437 We next discuss the removal of redundant ratios using a maximum spanning tree that simultaneously

438 maximizes log-ratio variance. After this, we introduce our network-based approach to feature selection 
and the two multivariate test statistics utilized to measure the strength of the association. We then

440 detail our between-group association maximization algorithm with pseudocode. Finally, we describe the

441 approach for assessing statistical significance via permutation testing using Monte Carlo sampling.

443 For a given metagenomic study, let $\mathbf{M} \in \mathbb{R}^{n x d}$ be the taxa count table for $\mathrm{n}$ samples and $d$ taxa. Here

444 we are interested in computing all $p=\frac{d(d-1)}{2}$ pairwise log ratios (PLR) of M. However, we must first

445 address the problem of zero counts. While there are numerous strategies with various drawbacks to

446 model and impute zeros [24,58-59] based on type/cause, there is in general no consensus of which

447 strategy should be used in metagenomic data. Notwithstanding, here we treat zero taxa counts as being

448 below the detection level, and we adopt a corresponding multiplicative replacement strategy for

449 imputing zeros proposed in [24] that preserves the essential log ratio and covariance structure. We

450 apply the closure operator to $\mathbf{M}$ to map the count data onto the unit-sum simplex where we define the

451 matrix $\mathbf{X}$ with elements $x_{i j}$ as

$$
x_{i j}=(C[\boldsymbol{M}])_{i j}=\frac{m_{i j}}{\sum_{k=1}^{d} m_{i k}} .
$$

452 We then set $\delta$ to be a constant equal to the smallest nonzero value across all of $\mathbf{X}$ and impute zeros to 453 obtain $\mathbf{R}$ with elements

$$
\mathrm{r}_{\mathrm{ij}}=\left\{\begin{array}{cl}
\delta & , x_{i j}=0 \\
x_{i j}\left(1-\delta \sum_{k=1}^{d} \mathbf{1}_{x=0}\left(\mathrm{x}_{i k}\right)\right), & x_{i j}>0
\end{array} \text { for }(i=1, \ldots, n)\right.
$$

454 We then compute all PLR from $\mathbf{R}$ to obtain $\mathbf{Z} \in \mathbb{R}^{n \times p}$ where $p=\frac{d(d-1)}{2}$ indicates the number of PLRs

455 (up to a sign). Because feature selection is critical to maximizing power and identifying sparse signals

456 hidden within noisy high-dimensional data, we seek to reduce the dimensionality through feature 
457 selection. Notably, this setting is distinct from traditional log ratio analysis [21] where dimensionality

458 reduction using PCA is applied to PLR/CLR transformed features to reduce dimensionality. Importantly,

459 the set of $p=\frac{d(d-1)}{2}$ different PLR are not independent of one another and require careful treatment

460 to select ratios that are independent of each other. Here we propose Differential Compositional

461 Variation (DCV), a scoring measure that enables efficient screening and ranking of PLR features within

462 compositional data. Similar to the screening concept in [60] for ultrahigh dimensional feature spaces,

463 DCV is motivated by Aitchison's compositional variation array [21] where patterns of compositional

464 variability for a group of data can be expressed in terms of the log ratio means $\xi_{j}=E\left[\mathbf{Z}_{*, j}\right]$ and

465 variances $\tau_{j}=\operatorname{var}\left(\mathbf{Z}_{*, j}\right)$ where $(j=1, \ldots, p)$. Similarly, here we define the logratio medians to be $\tilde{\xi}_{j}=$

$466 \tilde{\mathbf{Z}}_{*, \mathrm{j}}$. Our DCV score utilizes a diverse array of 5 statistics to score the contained variation of each log

467 ratio. Each component of DCV provides unique insight, thereby enabling efficient screening of

468 uninformative log ratios for downstream multivariate analysis. Let $y$ contain the labels for the binary

469 classes/groups $c_{1}$ and $c_{2}$ under consideration. In terms of $\xi_{j}$ and $\tau_{j}$, the first component of DCV, which

470 measures differences in group means, is Welch's t-statistic defined here as

471

$$
\Delta_{j}^{1}=\frac{\xi_{j}^{c_{1}}-\xi_{j}^{c_{2}}}{\sqrt{\left(\frac{\tau_{j}^{c_{1}}}{N_{x}}\right)+\left(\frac{\tau_{j}^{c_{2}}}{N_{y}}\right)}} .
$$

472 Next, we decompose the compositional variability of each $\mathrm{Z}_{*, j}$ using the classical F-statistic to again

473 measure means of differences:

474

$$
\Delta_{j}^{2}=\frac{n_{c_{1}}\left(\xi_{j}^{c_{1}}-\xi_{j}\right)^{2}+n_{c_{2}}\left(\xi_{j}^{c_{2}}-\xi_{j}\right)^{2}}{\tau_{j}^{c_{1}}+\tau_{j}^{c_{2}}}
$$


475 The third component of DCV, which measures heterogeneity of variances, is the Brown-Forsythe F-

476 Statistic. Here we define the jth $\log$ ratio to be the vector $\mathbf{z}=\mathbf{Z}_{*, \mathrm{j}}$. Let $x_{c j} \in \mathbf{z}$ where $\mathrm{c}$ and $\mathrm{j}$ denote the

477 jth observation of the cth group. From this, let $b_{c j}=\left|x_{c j}-\tilde{\xi}_{j}\right|$

478

$$
\Delta_{j}^{3}=\frac{\sum_{c} n_{c}\left(\bar{b}_{c \cdot}-\bar{b} . .\right)^{2}}{\sum_{c} \sum_{j}\left(b_{c j}-b_{c \cdot}\right)^{2} / \sum_{c}\left(n_{c}-1\right)},
$$

479 where $b_{c \cdot}=\sum \frac{b_{c j}}{n_{c}}$ and $\bar{b} . .=\sum \sum b_{c j} / \sum n_{c}$.

480 For the fourth component, we first define the empirical distribution function for each ordered log ratio $481 \mathrm{Z}_{*, j}$ to be

$$
F_{j}^{c}(x)=\frac{1}{n_{c}} \sum_{k=1}^{|c|} I_{\{-\infty, x\}} \mathrm{Z}_{k, j}^{c} \text { for each }(j=1, \ldots, p) \text { where } I_{\{-\infty, x\}}= \begin{cases}0, & \mathrm{Z}_{k, j}^{c}>x \\ 1, & \mathrm{Z}_{k, j}^{c} \leq x\end{cases}
$$

and from this, we set the fourth component equal to the Kolmogorov-Smirnov statistic by

$$
\Delta_{j}^{4}=\sup _{x}\left|F_{j}^{c_{1}}-F_{j}^{c_{2}}\right|
$$

The final component of DCV measures the information gain ratio which captures the ratio between the 486 amounts of entropy reduction when splitting the $j$ th log ratio, as implemented using the $\mathrm{R}$ fselectorCpp 487 package with default settings:

$$
\boldsymbol{\Delta}_{j}^{5}=\frac{H(\text { Group })+H\left(\mathbf{z}_{*, j}\right)-H\left(\operatorname{Group}, \mathbf{z}_{*, j}\right)}{H\left(\operatorname{Group}, \mathbf{z}_{*, j}\right)}
$$

489 where $H(X)=-P\left(x_{i}\right) \log \sum P\left(x_{i}\right)$ is Shannon's entropy and $H(X, Y)=H(X \mid Y)=\sum p(x, y) \log \frac{p(x, y)}{p(x)}$

490 is conditional Shannon's entropy. Aggregating these different components, we define the DCV matrix

$491 \quad \mathbf{V}=\left[\begin{array}{ccc}\Delta_{1}^{1} & \cdots & \Delta_{1}^{5} \\ \vdots & \ddots & \vdots \\ \Delta_{p}^{1} & \cdots & \Delta_{p}^{5}\end{array}\right]$. To account for differences in scale between the DCV components, we Z-score

492 standardize each score vector (column) to define the standardized DCV matrix $\widehat{\mathbf{V}}$ as 


$$
\hat{v}_{i j}=\frac{\mathrm{v}_{i j}-\bar{v}_{*, j}}{\mathrm{SD}\left(\mathrm{v}_{*, j}\right)} .
$$

494 The final set of DCV scores, $\widetilde{\mathbf{V}} \in \mathbb{R}^{p \times 1}$, which contains a score for each log ratio, is then defined as

$$
\check{v}_{j}=\sum_{k=1}^{5} \hat{v}_{j k}, \text { where }(j=1, \ldots, p)
$$

\section{Construction of DCV network and conversion to maximum spanning tree}

497 Here we leverage the inherent network structure of log ratios [27] to form our DCV network, defined as 498 a directed graph where edges point from numerator vertices to denominator vertices. We then define $499 G=(V, E, W)$ to be the DCV network where $V$ is the set of $d$ taxa vertices, $E$ is the edge set formed by 500 all $p$ pairwise log ratios formed between taxa vertices, and edge weights $W$ are the corresponding DCV 501 scores in $\overline{\mathbf{V}}$ between classes. In the initial phase of feature selection on $\mathbf{Z}$, we require the log ratio 502 subsets to meet three important properties: 1) explain maximum log ratio variance, 2) form a linearly 503 independent set, and 3) contain maximum DCV. Notably, the column rank of $\mathbf{Z}$ is $(d-1)$ and thus the 504 initial subset need only be a connected network containing all $d$ taxa to explain $100 \%$ of the log ratio 505 variance contained in $\mathbf{Z}$. The second property requires the undirected log ratio subset to be acyclic, as 506 may be achieved with a spanning tree; however, the number of spanning trees from $G$ can be expressed

507 by Cayley's formula: $T_{|V|}=|V|^{(|V|-2)}$. To circumvent considering this unmanageable number of 508 spanning trees, we utilize the weights imposed from the DCV scoring to enable efficient selection of a 509 suitable spanning tree from $G$, as described next.

510 To simultaneously meet all properties described above, we compute a Maximum Spanning Tree (MST), 511 containing all vertices without cycles and with maximum edge weight (among spanning trees. To make 512 this computationally tractable, we first sort the log ratios of $\widetilde{\mathbf{V}}$ in descending order by DCV score to form $513 \overline{\mathbf{V}}^{\prime}$ and retain the first set of $q$ log ratios that contain all $d$ taxa to form $\overline{\mathbf{V}}^{\prime \prime}$. We then define the log ratio 
514 network $G=(V, E, W)$ where $V$ is the set of $d$ taxa vertices, $E$ is the edge set of these $q$ pairwise log

515 ratios, with edge weights $W$ in $\breve{\mathbf{V}}^{\prime \prime}$. In practice, we have always found that the resulting networks at this

516 stage are connected - in the event that the network is not a single connected component, additional

$517 \log$ ratios from $\overline{\mathbf{V}}^{\prime}$ should be added to make it connected. Finally, from $G$ we compute the MST tree $G_{M S T}$

518 using the $\mathrm{R}$ igraph package. Selecting from $\mathbf{Z}$, we define $\mathbf{Z}^{\prime} \in \mathbb{R}^{n x(d-1)}$ to be the subset of log ratios

519 corresponding to the edge set of $G_{M S T}$.

Multivariate Test Statistics

521 SelEnergyPerm considers two multivariate test statistics to determine the statistical significance of 522 retained subsets of log ratios.

523 The first multivariate test statistic, the Distance Components F-ratio (discoF) is utilized when between-

524 group dispersion effects are not detected in Z'. The discoF statistic was proposed in [62] and is similar to 525 the traditional ANOVA ' $F$ ' ratio (but does not follow an F-distribution) where the total dispersion is 526 partitioned into between- and within-group components derived from an inter-sample Euclidean 527 distance matrix computed from Z'. Computation of the discoF statistic is done here using the energy R 528 package. As described in [62] the discoF test statistic, for binary groups is of the form

$$
F_{n, \alpha}=\frac{S_{n, \alpha}}{W_{n, \alpha} /(n-2)}
$$

530 where $S_{n, \alpha}$ is the between-sample energy statistic, $W_{n, \alpha}$ is the within-sample dispersion statistic and $0<$ $531 \alpha \leq 2$ is the exponent on the pairwise between sample norm. See $[37,62]$ for specific details on 532 computing the between- and within-group components of the discoF statistic. Here we use the energy $R$ 533 package to compute the discoF statistic.

534 The second statistic used by SelEnergyPerm is a combined- $F(c F)$ statistic which is distribution-free and 535 attempts to jointly account for differences in both location and scale between distributions. 
SelEnergyPerm selects the combined-F statistic when dispersion effects between groups are detected in

$537 \quad Z^{\prime}$. In particular, the SelEnergyPerm unscaled $c F$ statistic is the sum of F-ratios obtained from the PERMDISP2 with spatial medians [48] and PERMANOVA [35] procedures, computed using the R vegan package. In terms of the distances in $\mathbf{D}$, we partition the variation of $\mathbf{Z}^{\prime}$ and define the combined-F

540 statistic as

$$
c F=F_{\text {location }}+F_{\text {dispersion }}=\left(\frac{S S_{a}}{S S_{w} /(n-2)}\right)+\left(\frac{S S_{T}}{S S_{E} /(n-2)}\right)
$$

541 where $S S_{a}$ and $S S_{T}$ are the between-group sum of squares components and $S S_{w}$ and $S S_{E}$ are the

542 within-group sum of square components of variation from the PERMANOVA ( $\left.F_{\text {location }}\right)$ and PERMDISP2

$543\left(F_{\text {dispersion }}\right)$ procedures, respectively. See [35] and [48] for specific details on computing these

544 between- and within-group components. Likewise, the scaled combined-F $(\widehat{c F})$ statistic, a point

545 estimate, is computed in the same way but with z-score standardizing relative to permutation

546 distribution. Let $\boldsymbol{n} \boldsymbol{F}_{l o c}$. and $\boldsymbol{n} \boldsymbol{F}_{\text {disp. }}$. be $m$-dimensional vectors of null $F_{l o c}$. or $F_{d i s p .}$ statistics sampled

547 from the permutation distribution. We then scale $\widehat{F}_{\text {loc. }}=\frac{F_{\text {loc. }}-E\left[\boldsymbol{n} \boldsymbol{F}_{\text {loc. }]}\right.}{S D\left(\boldsymbol{n} \boldsymbol{F}_{\text {loc. }}\right)}$ and $\widehat{F}_{\text {disp. }}=\frac{F_{\text {disp. }}-E\left[\boldsymbol{n} \boldsymbol{F}_{\text {disp. } .}\right.}{S D\left(\boldsymbol{n} \boldsymbol{F}_{\text {disp. }}\right)}$.

$548 \quad$ Finally, we define

$$
\widehat{c F}=\widehat{F}_{\text {loc. }}+\widehat{F}_{\text {disp }}
$$

550 Of note, $\widehat{C F}$ is approximate and thus the estimate has variability based on the number of samples drawn

551 from the permutation distribution. We consider $m=1 \mathrm{e} 6$ samples here as a balance between

552 computational cost and minimizing this variation.

554 In this step, we focus on the multivariate structure formed by a subset of log ratios. Specifically, we are 555 interested in maximizing the between-group variation induced by a subset of log ratios in a low-

556 dimensional multivariate space. To find a minimal, statistically significant subset of log ratios that 
557 maximizes $F_{n, \alpha}$ (location effects only) or $c F$ (dispersion and location effects) between classes, we utilize

558 a greedy forward stepwise feature selection procedure.

\section{Algorithm 1 Association maximization with greedy forward stepwise selection}

1: $\quad$ Procedure selectionEnergy $\left(\mathbf{Z}^{\prime}, \mathbf{y}\right.$, eps $=1 \mathrm{e}-5$, patience $\left.=25, \alpha=0.05\right)$

2: $\quad$ if PERMDISP2 $\left(\mathbf{Z}^{\prime}, \mathbf{y}\right)<\alpha$ then

Det. test statistic

3: $\quad$ testStatisticFunction $=c F()$

4: $\quad$ Metric $=$ 'combinedF'

5: else

6: $\quad$ testStatisticFunction $=F_{n, \alpha}()$

7: $\quad$ Metric $=$ 'discoF'

8: $\quad$ end if

9: $\quad \mathbf{X}=\mathbf{Z}^{\prime}[, 1: 3]$

$\triangle$ Start with top 3 LRs

10: $\operatorname{maxF}=$ testStatisticFunction(baseSet,y)

11: $\quad$ improvementTime $=0$

12: for $x$ in $4, \ldots$, numCols $\left(Z^{\prime}\right)$ do

13: $\quad \mathbf{X}_{\text {new }}=\mathbf{X} \cup \mathbf{Z}^{\prime}[, \mathbf{x}]$

14: $\quad$ newF $=$ testStatisticFunction(newSet,y)

15: $\quad$ diff $=$ newF - maxF

16: if diff $\geq$ eps then

17: $\mathbf{X}=\mathbf{X}_{\text {new }}$

18: $\operatorname{maxF}=$ newF

19: end if

20: $\quad$ if diff $\geq$ eps then 
21: $\quad$ improvementTime $=0$

22: else

23: $\quad$ improvementTime $=$ improvementTime +1

24: end if

25: if improvementTime $>$ patience then

26: Break

27: $\quad$ end if

28: end for

29: if Metric $==$ 'discoF' then

30: $\quad$ testStat $=F_{n, \alpha}() \mid \mathbf{X}$

31: else

32: $\quad$ testStat $=\widehat{c F} \mid \mathbf{X}$

33: end if

34: return list( $\mathbf{X}$, testStat )

35: end procedure

561 To assess the statistical significance of the association $F^{o b s}=\operatorname{selectionEnergy}\left(\mathbf{Z}^{\prime}, \mathbf{y}\right)$ [testStat]

562 computed on the log-ratio subset $\mathbf{S}=\operatorname{selectionEnergy}\left(\mathbf{Z}^{\prime}, \mathbf{y}\right)[\mathbf{X}]$ returned from Algorithm 1, we utilize

563 permutation testing via Monte Carlo sampling [63]. Specifically, we test if the $F^{\text {obs }}$ is more extreme than

$564 \quad F$-ratios sampled from the permutation distribution $\left(F^{n u l l}\right)$. In particular, log-ratio subsets and

565 association statistics are computed using Algorithm 1 with permuted labels $(\boldsymbol{y})$. With $k$ random samples

566 of $\boldsymbol{F}^{\text {null }}$ the one-sided estimated exact p-value becomes 


$$
\hat{p}=\frac{1+\sum_{i=1}^{k} I\left(f_{i}^{\text {null }}>F^{o b s}\right)}{k+1}
$$

\section{Simulation Strategy}

We adapted several simulation settings to investigate and highlight key association detection characteristics of SelEnergyPerm when compared to ANOSIM, PERMANOVA, and the energy test.

571 Additionally, to detect the presence of heterogeneity of multivariate dispersion between groups and

572 understand its impact on association detection, we utilized the PERMDISP2 method as an indicator. The

573 empirical association detection ability of each method was assessed within a binary classification

574 framework. To do this, we measured the rate of each statistical test to correctly reject (Power) or accept

575 (Type I Error) the null hypothesis (no difference between groups) at significance $\alpha=0.05$. Further, to

576 truly assess detection capabilities, we presented each method with binary instances drawn from either

577 the same (Null Case) or different (True Case) distributions for each scenario using Monte Carlo

578 simulations. The Matthews Correlation Coefficient (MCC), which effectively summarizes the binary

579 confusion matrix, was then used to measure the overall accuracy of each method's ability to detect

580 associations across various simulation scenarios. MCC was computed as

581

$$
\mathrm{MCC}=\frac{(T P * T N)-(F P * F N)}{\sqrt[2]{(T P+F P)(T P+F N)(T N+F P)(T N+F N)}}
$$

where TP = true positive (reject the null hypothesis for True Case), TN = true negative (accept null

hypothesis for Null Case), FP = false positive (reject the null hypothesis for Null Case), FN = false negative

584 (accept null hypothesis for True Case). For each simulation scenario, we generated 100 simulated datasets with 40 samples in both Class $1\left(S_{1}\right)$ and Class $2\left(S_{2}\right)$ for the balanced binary design and 20/60

586 (Class 1 / Class 2) samples for the unbalanced design for each case. Given all methods rely on

587 permutation testing for significance, we generate a common set of 150 permutations per dataset to consistently compute significance for each method across all scenarios and settings. 


\section{Simulation Scenarios (Synthetic Data)}

590 For all synthetic data scenarios, we consider datasets with $d=50,150,250$ dimensions, yielding a total 591 of $p=1225,11175,31125$ pairwise log ratios, respectively. Each of the following simulation scenarios

592 is available in our SelEnergyPermR R package available at

593 https://github.com/andrew84830813/selEnergyPermR using the function scenarioN() where $N=[1,5]$.

594 In Scenario 1, for the true case, we consider both multivariate location (in all dimensions) and dispersion 595 effects that grow with increased dimensions. The increase in dispersion with dimension is similar to 596 settings studied in [42]. Here, data from each sample arise from the Dirichlet distribution $\operatorname{Dir}(\boldsymbol{\alpha})$

597 whereby data are naturally constrained within the unit-sum simplex and are commonly used to model 598 compositional data. Data from class 1 are simulated with $\alpha_{1}=(3,3, \ldots, 3)$. Data from class 2 are 599 generated with $\alpha_{2}=\left(3 \cdot \frac{\log (d)}{5}, 3 \cdot \frac{\log (d)}{5}, \ldots, 3 \cdot \frac{\log (d)}{5}\right)$ where the $\frac{\log (d)}{5}$ term shifts the overall 600 location and increases dispersion as the dimensionality increases. For the null case, data from both 601 classes are generated from $\operatorname{Dir}\left(\alpha_{1}\right)$.

602 In Scenario 2, for the true case, we generate sparse count data from two Dirichlet distributions that 603 differ in the location of the first component and overall dispersion. Library size or total counts for the ith 604 sample were modeled using a negative binomial (NB) distribution and simulated as $C_{i} \sim N B\left(s, \frac{s}{s+\mu}\right)$ 605 where $s=1$ and $\mu=1 \mathrm{e} 7$. Count data for class 1 were generated by rounding:

$606 C_{i} * \operatorname{Dir}\left(\boldsymbol{\alpha}_{1}\right)$ where $\boldsymbol{\alpha}_{1}=\left(U_{1} \sim\right.$ unif $(3000,5000), U_{i \in[2,10]} \sim$ unif $(500,1500), U_{i \in[11, d]} \sim$ unif $\left.(1,5)\right)$

607 Count data for class 2 were generated as:

$C_{i} * \operatorname{Dir}\left(\alpha_{1}\right)$ where $\alpha_{1}=\left(U_{1} \sim\right.$ unif $(12500,17500), U_{i \in[2,10]} \sim$ unif $(500,1500), U_{i \in[11, d]} \sim$ unif $\left.(1,5)\right)$

609 Notably, the $U_{i \in[11, d]} \sim$ unif $(1,5)$ terms are used to model sparsity. For the null case, data from both 610 classes are generated from $C_{i} * \operatorname{Dir}\left(\alpha_{1}\right)$ vector. 
611 In Scenario 3, for the true case, we generate compositional data whereby there exists a large location

612 effect that increases while the dispersion effects decrease with dimensionality. These settings are

613 similar to settings considered for association benchmark comparisons in [42]. In particular, we simulate

614 data from the additive logistic normal distribution on the simplex [21]. To do this we first let $\boldsymbol{S}_{\mathbf{1}}=$

$615 N\left(\boldsymbol{\mu}_{1}, \boldsymbol{\Sigma}_{1}\right)$ and $\boldsymbol{S}_{2}=N\left(\boldsymbol{\mu}_{2}, \boldsymbol{\Sigma}_{2}\right)$ be samples drawn from multivariate normal distributions. We set $\mu_{1}=$

$616(0,0, \ldots, 0)$ and $\mu_{2}$ to be equal to $1 / \sqrt{d}$ in the first $25 \%$ of dimensions and 0 in the remaining dimensions.

617 The covariance structure was defined in the same way as [42] where $\Sigma$ was defined with 1's along the

618 main diagonal and 0.2 along the two diagonals off the main. From this, $\boldsymbol{\Sigma}_{1}=\boldsymbol{\Sigma}+\delta \boldsymbol{I}_{d}$ and $\boldsymbol{\Sigma}_{2}=\boldsymbol{\Sigma}+\boldsymbol{U}+$

$619 \delta \boldsymbol{I}_{d}$ where $\boldsymbol{U}$ is a $d x d$ matrix with unif $\left(0,32 / d^{2}\right)$ entries and $\delta=$

$620 \mid \min ($ eigenvalues $(\boldsymbol{\Sigma})$, eigenvalues $(\boldsymbol{\Sigma}+\boldsymbol{U})) \mid+0.05$. Here row vectors from $\boldsymbol{S}$ represent additive log-

621 ratio (ALR) vectors and are subsequently projected onto the simplex using the inverse additive log-ratio

622 transformation defined as $\mathrm{ALR}^{-1}=\mathrm{C}(\exp (\boldsymbol{s}))$. For the null case, data for both classes were simulated

623 from $N\left(\boldsymbol{\mu}_{1}, \boldsymbol{\Sigma}_{1}\right)$.

624 In Scenario 4, for the true case, we generate compositional data where there exist sparse location

625 effects in the first dimension that grow stronger while dispersion effects grow weaker as the

626 dimensionality increases. That is, $\boldsymbol{S}_{\mathbf{1}}=N\left(\boldsymbol{\mu}_{1}, \boldsymbol{\Sigma}_{1}\right)$ and $\boldsymbol{S}_{\mathbf{2}}=N\left(\boldsymbol{\mu}_{2}, \boldsymbol{\Sigma}_{2}\right)$ are defined as in scenario 3

627 except for $\mu_{2}$ being set to $\log \left(\frac{d}{3}\right)$ in the first dimensions and 0 in the remaining dimensions. The simplex

628 projection and null case are done as described in scenario 3.

629 Finally, in Scenario 5 for the true case, we generate compositional data from the additive logistic normal

630 distribution with a small location shift and large dispersion difference that increases with dimensionality.

631 Let $\boldsymbol{S}_{\mathbf{1}}=N\left(\mu_{1}, \Sigma_{1}\right)$ and $\boldsymbol{S}_{2}=N\left(\mu_{2}, \Sigma_{2}\right)$ be defined in as in scenario 3 except for $\mu_{2}$ set to $\frac{1}{\sqrt{n_{1}+n_{2}}}$ in all

632 dimensions and $\boldsymbol{U}$ now being a $d x d$ matrix with unif $(0,32)$. The simplex projection and null case are

633 done as described in scenario 3. 


\section{Simulation Scenarios (Experimental Data)}

635 For all experimental data scenarios, we used publicly available taxa count tables where sequencing data were already pre-processed. Each of the following simulation scenarios are available in our

637 SelEnergyPermR R package available at https://github.com/andrew84830813/selEnergyPermR using the 638 functions simFromExpData.covarianceShift() or simFromExpData.largeMeanShft().

639 For the 16S data characteristics, we utilized the ob_goodrich_results.tar.gz dataset from the

640 microbiomeHD $[64,65]$ database. We then aggregated the taxa to the genus level (distinct genera $=247)$

641 and extracted the 428 healthy samples from the goodrich16S dataset for our 165 data simulations. For

642 WGS data characteristics, we utilized the ZeeviD2015 [66] dataset from the curatedmetagenome [67]

643 database. We aggregated taxa counts by species (distinct species $=1,776$ ) and then extracted the 900

644 control samples for our WGS data simulations. Here we model the $16 S$ and WGS count data using zero-

645 inflated negative binomial (ZINB) models which have been shown to be a reasonable choice for

646 modeling microbiome count data [68]. ZINB models were fit to the 165 and WGS dataset described

647 above using the ZINBWAVE R package and default settings. For all experimental data scenarios, we used

648 the fitted 16S/WGS ZINB models to simulate new samples for each dataset. That is, we first simulated 649428 samples from the ZINB model for the 16 S datasets or 900 samples for the WGS datasets. We then

650 randomly select 40 samples per class $\left(S_{1}\right.$ and $\left.S_{2}\right)$ for the balanced design and 20/60 $\left(S_{1}\right.$ and $\left.S_{2}\right)$ samples

651 for the unbalanced design. To reduce the presence of rare features we only retained features present in 652 at least $15 \%$ of all samples for all datasets.

653 For Scenario 1, we consider for the true case in both $16 \mathrm{~S}$ and WGS datasets, settings where the percent

$654 P=(5,20,35,50)$ of dimensions with a location shift increases while the dispersion effect between

655 classes remains fixed. To do this, we first simulate count data $\mathbf{X}$ from the ZINB model and map it onto 
the unit-sum simplex using Eq.1 and impute zeros using Eq.2. The ALR transformation is then applied to

$657 \quad \boldsymbol{X}$ as

658

$$
x_{i j}=\ln \left(\frac{x_{i j}}{x_{i p}}\right), j \neq p \text { and } p=d
$$

Using the ALR-transformed data we define $\boldsymbol{Z}=N(\boldsymbol{\mu}, \boldsymbol{\Sigma})$ where

$$
\boldsymbol{\mu}=\mathrm{E}[\boldsymbol{X}]=\left(\mathrm{E}\left[X_{1}\right], \mathrm{E}\left[X_{2}\right], \ldots \mathrm{E}\left[X_{d-1}\right]\right)^{T}
$$

660 and

$$
\Sigma_{\mathbf{i j}}=\operatorname{cov}\left[X_{i}, X_{j}\right]
$$

661 The variance ( $\operatorname{diag}(\boldsymbol{\Sigma})$ ) of each dimension is ranked in ascending order whereby $\boldsymbol{\mu}$ and $\boldsymbol{\Sigma}$ are reordered 662 accordingly to form $\boldsymbol{\mu}_{\boldsymbol{r}}$ and $\boldsymbol{\Sigma}_{\mathbf{r}}$. Of note, this is done to ensure the location shift occurs in features with 663 minimal variance. We then simulate $\boldsymbol{S}_{\mathbf{1}}$ from $N\left(\boldsymbol{\mu}_{\boldsymbol{r}}, \boldsymbol{\Sigma}_{\mathbf{r}}\right)$ and define $\boldsymbol{\mu}_{\mathbf{1}}$ and $\boldsymbol{\Sigma}_{\mathbf{1}}$ using Eqs.4-5. Letting $664 \boldsymbol{\mu}_{2}=\boldsymbol{\mu}_{1}$ we then shift the first $P_{i} \%$ of dimensions of $\boldsymbol{\mu}_{\mathbf{2}}$ by a factor of 1.25 . From this we simulate $\boldsymbol{S}_{2}$ 665 from $N\left(\boldsymbol{\mu}_{2}, \boldsymbol{\Sigma}_{\mathbf{1}}\right)$. Finally, $\boldsymbol{S}_{\mathbf{1}}$ and $\boldsymbol{S}_{\mathbf{2}}$, which are in Euclidean ALR form, are mapped back to the simplex 666 (relative abundance) using the inverse ALR transformation. For the null case data for both classes are 667 simulated from $N\left(\boldsymbol{\mu}_{r}, \boldsymbol{\Sigma}_{\mathbf{r}}\right)$.

668 Finally, for Scenario 2, we consider for the true case in both $16 \mathrm{~S}$ and WGS datasets, settings with a 669 location shift in the first $10 \%$ of dimensions confounded by increasing dispersion effects. Here we 670 compute $\boldsymbol{S}_{\mathbf{1}}$ in Euclidean ALR form as described in Scenario 2 (Experimental Data) such that $671 \boldsymbol{S}_{1} \sim N\left(\boldsymbol{\mu}_{1}, \boldsymbol{\Sigma}_{\mathbf{1}}\right)$. From this, $\boldsymbol{\Sigma}_{s_{1}}=\boldsymbol{\Sigma}_{1}+\delta \boldsymbol{I}_{d}$ and $\boldsymbol{\Sigma}_{s_{2}}=\boldsymbol{\Sigma}_{1}+\boldsymbol{U}+\delta \boldsymbol{I}_{d}$ where $\boldsymbol{U}$ is a $d x d$ matrix with 672 unif $\left(0, \beta_{\mathrm{i}}\right)$ entries and $\delta=\mid \min ($ eigenvalues $(\boldsymbol{\Sigma})$, eigenvalues $(\boldsymbol{\Sigma}+\boldsymbol{U})) \mid+0.05$. For 16S data $\beta=$ $673(0.10,1.40,2.70,4.00)$ and for WGS data $\beta=(0.10,4.07,8.03,12.00)$. Additionally, letting $\boldsymbol{\mu}_{2}=\boldsymbol{\mu}_{1}$ we 674 shift the first $10 \%$ of dimensions of $\boldsymbol{\mu}_{2}$ by a constant factor of 1.25 for WGS data and by a factor $F=$ $675(1.20,1.17,1.13,1.10)$ for $16 \mathrm{~S}$ data. In all, the final multivariate forms are $\boldsymbol{S}_{1} \sim N\left(\boldsymbol{\mu}_{1}, \boldsymbol{\Sigma}_{S_{1}}\right)$ and 
$676 \boldsymbol{S}_{1} \sim N\left(\boldsymbol{\mu}_{2}, \boldsymbol{\Sigma}_{S_{2}}\right)$. These distributions, which are in ALR form, are mapped back onto the simplex using the

$677 \mathrm{ALR}^{-1}$ transform. Lastly, for the null case, data for both classes are simulated from $N\left(\boldsymbol{\mu}_{1}, \boldsymbol{\Sigma}_{S_{1}}\right)$.

678 Feature Selection Benchmarks

679 For the feature selection (FS) benchmark we used the Boruta R package with maxRun set to 100 and 680 importance set to Gini for the Boruta FS. The glmnet R package was used for LASSO FS where alpha was 681 set to 1 and lambda was optimized via cross-validation. The caret R package was used to implement RFE 682 FS where 5-fold cross-validation was used to evaluate AUC and feature importance of sets $s=$ $683\left(2^{1}, 2^{2}, \ldots, 2^{n}\right)$, where $n=$ floor $\left(\log _{2} p\right)$. The FSelectorRcpp R package with default settings was used 684 for the Information Gain Filter FS. For each Scenario (Synthetic Data) FS characteristics were evaluated 685 on 200 synthetic datasets across feature space sizes of $p=(1225,4950,11175,19900,31125) \log$ ratios. Performance characteristics considered were the number of log ratios selected, log-ratio network 687 clustering coefficient, and the combined-F statistic. Here we use the number of log ratios selected by 688 each method as a proxy for model complexity. Specifically, higher model complexity or the number of 689 features retained increases the risk of overfitting and unnecessarily reduces the biological interpretation corresponding to the log ratios. Log-ratio networks were formed using the final subset selected by each

691 method, defined as a graph where vertices represent taxa and edges connect taxa pairs to form a log 692 ratio. Redundancy in a log-ratio network of this type can be inferred from cycles in the network. While it does not detect all cycles, the clustering coefficient can be used here to detect cycles between three

694 nodes (closed triangles versus triplets). Computation of the global clustering coefficient was done using 695 the R igraph package. Finally, the $c F$ statistic, which measures the strength of the overall association 696 was computed as in Eq. 3 for each subset. All performance characteristics were evaluated in both 697 balanced and unbalanced sampling designs. Computational time was recorded in seconds for each 698 simulation scenario, feature space, and sample design. The recorded time represents the CPU time 699 required by each FS method to select the final log ratio subset. All computations were run on UNC- 

with $5 G B$ of RAM.

\section{Discussion}

We presented SelEnergyPerm, a group association testing framework for high-dimensional

metagenomic data with sparse microbiome associations between groups. Our framework directly

accounts for the compositional sample space imposed on these data as a result of technical variations in sample-wise library size $[17,19]$. This is done by using embedded feature selection on a set of robust all

707 pairwise log ratios to improve the detection and interpretation of sparse signals hidden in these data.

708 Each log ratio is first ranked using our novel DCV score followed by the application of network methods 709 and feature selection techniques to effectively select subsets of log ratios. In tandem, these steps help

710 to identify log ratio signatures capable of explaining microbiome-derived phenotypic differences.

711 Further, false discovery is properly controlled for by repeating the entire process with permuted labels

712 in unrestricted or restricted single or restricted permutation test designs for statistical significance [64].

713 We assessed our method by conducting an extensive simulation study to rigorously benchmark the

714 performance of the method relative to popular alternatives for both feature selection and association

715 testing. Our simulation scenarios included data from both synthetic and empirical distributions where

716 scenarios included settings with small/large location shifts embedded in sparse/dense signals and

717 small/large location shifts with covariance differences embedded in sparse/dense association signals

718 carried out on both balanced and unbalanced sample designs. When compared to popular alternatives,

719 we show our SelEnergyPerm feature selection approach is, overall, able to select fewer log ratios,

720 guarantee log-ratio subsets are independent, and better maximize between-group associations with

721 relatively modest computational time requirements. Additionally, when compared to common

722 association testing methods used in metagenomic studies, we show SelEnergyPerm can consistently 
723 detect associations better than or comparable with the alternatives in nearly all simulation settings

724 tested. The better performance of SelEnergyPerm is most notable when sparse association signals are 725 present.

726 Our demonstration of how SelEnergyPerm can be used to gain robust and unique biological insight was

727 carried out in detail with data from 4 case studies. In the first case study, SelEnergyPerm successfully

728 detected a confirmed association between the composition of the microbiome in CSF and PIH/NPIH

729 disease status in Ugandan infants using a reduced log ratio signature (13 of 1,596 log ratios). Further, we

730 show, given these data that our log-ratio signature can to a greater degree discriminate between

731 disease statuses and explain differences between infants than with a single feature or all pairwise log

732 ratios. In our second case study, SelEnergyPerm detected an association between delivery mode and the 733 gut microbiome composition in infants during the first 2 months of life and at the time 0 collection time.

734 Notably, PERMANOVA and ANOSIM applied over the same time course with all log ratios failed to detect 735 this association. In the third case study, SelEnergyPerm detected an association between the 736 composition of the gut microbiome and abnormal fecal calprotectin levels. Here we found our fecal 737 calprotectin associated log-ratio signature (26 log ratios) had a comparable discriminatory ability to the 738 uninterpretable all log ratios set (7,381 log ratios), thus enabling easier biological interpretation. In the 739 final case study, SelEnergyPerm detected and characterized associations between the microbiome 740 composition in early life and the development of food allergy later in life.

741 Overall, our results demonstrate that SelEnergyPerm is a powerful framework for detecting sparse 742 association under various scenarios. However, in the presence of heterogeneity of variance and/or 743 unbalanced group designs, common enemies of multivariate association testing method, the power of 744 SelEnergyPerm was reduced, albeit to a lesser degree than the standard methods tested. Therefore, 745 caution should be used when applying SelEnergyPerm in these settings. Additionally, in some scenarios 746 with dense association signals, the performance of SelEnergyPerm was slightly reduced when compared 
747 to standard methods. While the power reduction was small, the enhanced interpretation from a smaller 748 log-ratio signature may outweigh the loss of power in some settings.

749 Notwithstanding these limitations, SelEnergyPerm is the first method to our knowledge to fully utilize 750 the pairwise log ratio compositional approach in a group association testing framework for 751 metagenomic data. Importantly, given the compositional sample space imposed on these data, where 752 features are relative, our approach enables the discovery of associations using pairwise log ratios which, 753 by design, robustly interpret features relative to one another rather than alone. While the benefits of 754 employing log ratios are well documented, implementing and carrying out these analyses can be 755 challenging and time-consuming in practice. To this end, we developed an R package SelEnergyPermR 756 with functions to perform the method presented in this paper. Additionally, our package enables rapid 757 pre-processing of relative abundance data, calculation of all pairwise log ratios, and multiplicative zero 758 imputation. Our package also includes functions to simulate data from all scenarios presented in this 759 work. Lastly, our approach adds to a small list of compositional methods for testing associations [30-32] and is to our knowledge, the first compositional data method developed for sparse multivariate group 761 association testing in metagenomic data. We also add to a small list of compositional approaches for 762 feature selection [69] however unlike these methods, our approach directly uses pairwise log ratios 763 which enables simple interpretation and may better elucidate taxa-taxa interactions through log ratio 764 network analysis.

765 We developed SelEnergyPerm to be a powerful group association test for researchers interested in 766 studying 165 or WGS microbiome data with a compositional approach using pairwise log ratios. While 767 not demonstrated explicitly here, SelEnergyPerm is also compatible with multiclass group association 768 testing. Future directions to usefully expand this methodology could focus on incorporating covariate 769 information and extending the framework to longitudinal data. 


\section{Ethics approval and consent to participate}

772 All datasets used in this manuscript are publicly available. No ethics approval or consent to participate

773 was required for this manuscript.

\section{Consent for publication}

775 All datasets used in this manuscript are publicly available. No ethics approval or consent to participate

776 was required for this manuscript.

\section{Availability of data and material}

778 Public metagenomic data used in the analysis presented in this paper can be directly accessed for (1)

779 Ugandan infant's cerebrospinal fluid microbiomes and post-infectious hydrocephalus

780 (https://microbiomedb.org/mbio/app/downloads/release-

$781 \quad 22 / 953 \mathrm{~b} 8 \mathrm{ff} 2 \mathrm{~d} 4 \mathrm{ba} 436 \mathrm{fa} 16 \mathrm{c} 381916 \mathrm{a} 57850 \mathrm{~b} 43 \mathrm{a} 2 \mathrm{a} 58$ ), (2) delivery mode and the composition of infant's

782 gut microbiomes over the first three months of life (https://qiita.ucsd.edu/; study ID = 10249), (3) adult

783 gut microbiomes and abnormal fecal calprotectin levels

784 (https://ibdmdb.org/tunnel/public/HMP2/WGS/1818/products ; taxonomic_profiles_3.tsv.gz, HMP2

785 Metadata), and (4) the gut microbiome composition of infants within the first 6 months of life and

786 future allergic sensitization to egg, milk, or peanuts

787 (https://diabimmune.broadinstitute.org/diabimmune/; Three Study Cohort). Data used in real 16S and 788 WGS data simulation were accessed from 16S (https://zenodo.org/record/1146764\#.YOc3KehKhEY;

789 ob_goodrich_results.tar.gz) and WGS (https://doi.org/doi:10.18129/B9.bioc.curatedMetagenomicData;

790 ZeeviD2015). 
791 The selEnergyPerm method has been implemented in a freely available R packaged at:

792 https://github.com/andrew84830813/selEnergyPermR.git. All analysis was done in R 4.0.5. Analysis R

793 script and input (publicly accessed data) and output (processed in this study) data can be accessed from:

794 https://github.com/andrew84830813/SelectionEnergyPerm Project.git

\section{Competing interests}

796 The authors declare that they have no competing interests.

797 Funding.

798 This research was funded by an HHMI Gilliam Award (GT11504) and a JSMF Complex Systems Scholar 799 Award (\#220020315). The content is solely the responsibility of the authors and does not necessarily 800 represent the official views of any agency funding this research.

\section{Authors' contributions}

802 ALH developed the framework methodology, performed simulations and case studies, developed the 803 software package and wrote the manuscript. PJM oversaw the development of the framework 804 methodology and wrote the manuscript. ALH and PJM approved the final manuscript.

805 Acknowledgments.

806 We acknowledge and are grateful to our collaborators for many conversations that helped motivate and 807 further refine this effort, including Jeff Henderson, Elise Hickman, Ilona Jaspers, Mike Kulis, Laura Marks, 808 and John Robinson.

809 References

810 1. Martín, R., Miquel, S., Langella, P., \& Bermúdez-Humarán, L. G. (2014). The role of metagenomics in 811 understanding the human microbiome in health and disease. Virulence, 5(3), 413-423.

812 https://doi.org/10.4161/viru.27864 
2. Ranjan, R., Rani, A., Metwally, A., McGee, H. S., \& Perkins, D. L. (2016). Analysis of the microbiome: Advantages of whole genome shotgun versus $16 \mathrm{~S}$ amplicon sequencing. Biochemical and Biophysical Research Communications, 469(4), 967-977. https://doi.org/10.1016/j.bbrc.2015.12.083

3. Gopalakrishnan, V., Helmink, B. A., Spencer, C. N., Reuben, A., \& Wargo, J. A. (2018). The Influence of the Gut Microbiome on Cancer, Immunity, and Cancer Immunotherapy. Cancer Cell, 33(4), 570580. https://doi.org/10.1016/j.ccell.2018.03.015

4. Manichanh, C., Borruel, N., Casellas, F., \& Guarner, F. (2012). The gut microbiota in IBD. Nature Reviews Gastroenterology \& Hepatology, 9(10), 599-608. https://doi.org/10.1038/nrgastro.2012.152

5. Turnbaugh, P. J., Ley, R. E., Mahowald, M. A., Magrini, V., Mardis, E. R., \& Gordon, J. I. (2006). An obesity-associated gut microbiome with increased capacity for energy harvest. Nature, 444(7122), 1027-1031. https://doi.org/10.1038/nature05414

6. Qin, N., Yang, F., Li, A., Prifti, E., Chen, Y., Shao, L., Guo, J., Le Chatelier, E., Yao, J., Wu, L., Zhou, J., Ni, S., Liu, L., Pons, N., Batto, J. M., Kennedy, S. P., Leonard, P., Yuan, C., Ding, W., ... Li, L. (2014). Alterations of the human gut microbiome in liver cirrhosis. Nature, 513(7516), 59-64. https://doi.org/10.1038/nature13568

7. Foster, J. A., \& McVey Neufeld, K.-A. (2013). Gut-brain axis: How the microbiome influences anxiety and depression. Trends in Neurosciences, 36(5), 305-312. https://doi.org/10.1016/j.tins.2013.01.005

8. Kong, H. H., Oh, J., Deming, C., Conlan, S., Grice, E. A., Beatson, M. A., Nomicos, E., Polley, E. C., Komarow, H. D., Murray, P. R., Turner, M. L., \& Segre, J. A. (2012). Temporal shifts in the skin microbiome associated with disease flares and treatment in children with atopic dermatitis. Genome Research, 22(5), 850-859. https://doi.org/10.1101/gr.131029.111 
9. Dewhirst, F. E., Chen, T., Izard, J., Paster, B. J., Tanner, A. C. R., Yu, W.-H., Lakshmanan, A., \& Wade, W. G. (2010). The human oral microbiome. Journal of Bacteriology, 192(19), 5002-5017. https://doi.org/10.1128/JB.00542-10

10. Wilson, M. T., \& Hamilos, D. L. (2014). The Nasal and Sinus Microbiome in Health and Disease. Current Allergy and Asthma Reports, 14(12), 485. https://doi.org/10.1007/s11882-014-0485-x

11. Zackular, J. P., Rogers, M. A. M., Ruffin, M. T., \& Schloss, P. D. (2014). The Human Gut Microbiome as a Screening Tool for Colorectal Cancer. Cancer Prevention Research, 7(11), 1112-1121. https://doi.org/10.1158/1940-6207.CAPR-14-0129

12. Schlaberg, R. (2020). Microbiome Diagnostics. Clinical Chemistry, 66(1), 68-76. https://doi.org/10.1373/clinchem.2019.303248

13. Caporaso, J. G., Kuczynski, J., Stombaugh, J., Bittinger, K., Bushman, F. D., Costello, E. K., Fierer, N., Peña, A. G., Goodrich, J. K., Gordon, J. I., Huttley, G. A., Kelley, S. T., Knights, D., Koenig, J. E., Ley, R. E., Lozupone, C. A., McDonald, D., Muegge, B. D., Pirrung, M., ... Knight, R. (2010). QIIME allows analysis of high-throughput community sequencing data. Nature Methods, 7(5), 335-336. https://doi.org/10.1038/nmeth.f.303

14. Schloss Patrick D., Westcott Sarah L., Ryabin Thomas, Hall Justine R., Hartmann Martin, Hollister Emily B., Lesniewski Ryan A., Oakley Brian B., Parks Donovan H., Robinson Courtney J., Sahl Jason W., Stres Blaz, Thallinger Gerhard G., Van Horn David J., \& Weber Carolyn F. (2009). Introducing mothur: Open-Source, Platform-Independent, Community-Supported Software for Describing and Comparing

15. Truong, D. T., Franzosa, E. A., Tickle, T. L., Scholz, M., Weingart, G., Pasolli, E., Tett, A., Huttenhower, C., \& Segata, N. (2015). MetaPhIAn2 for enhanced metagenomic taxonomic profiling. Nature Methods, 12(10), 902-903. https://doi.org/10.1038/nmeth.3589

16. Wood, D. E., Lu, J., \& Langmead, B. (2019). Improved metagenomic analysis with Kraken 2. Genome Biology, 20(1), 257. https://doi.org/10.1186/s13059-019-1891-0 
17. Gloor, G. B., Macklaim, J. M., Pawlowsky-Glahn, V., \& Egozcue, J. J. (2017). Microbiome Datasets Are Compositional: And This Is Not Optional. Frontiers in Microbiology, 8. https://doi.org/10.3389/fmicb.2017.02224

18. Gloor, G. B., \& Reid, G. (2016). Compositional analysis: A valid approach to analyze microbiome highthroughput sequencing data. Canadian Journal of Microbiology, 62(8), 692-703. https://doi.org/10.1139/cjm-2015-0821

19. Weiss, S., Xu, Z. Z., Peddada, S., Amir, A., Bittinger, K., Gonzalez, A., Lozupone, C., Zaneveld, J. R., Vázquez-Baeza, Y., Birmingham, A., Hyde, E. R., \& Knight, R. (2017). Normalization and microbial differential abundance strategies depend upon data characteristics. Microbiome, 5. https://doi.org/10.1186/s40168-017-0237-y

20. Pearson, K. (1897). Mathematical contributions to the theory of evolution.-On a form of spurious correlation which may arise when indices are used in the measurement of organs. Proceedings of the Royal Society of London, 60(359-367), 489-498. https://doi.org/10.1098/rspl.1896.0076

21. Aitchison, J. (1986). The Statistical Analysis of Compositional Data. London: Chapman \& Hall.

22. Greenacre, M., \& Lewi, P. (2009). Distributional Equivalence and Subcompositional Coherence in the Analysis of Compositional Data, Contingency Tables and Ratio-Scale Measurements. Journal of Classification, 26(1), 29-54. https://doi.org/10.1007/s00357-009-9027-y

23. Pawlowsky-Glahn, V., \& Buccianti, A. (2011). Compositional Data Analysis: Theory and Applications. John Wiley \& Sons.

24. Martín-Fernández, J.-A., Hron, K., Templ, M., Filzmoser, P., \& Palarea-Albaladejo, J. (2015). Bayesianmultiplicative treatment of count zeros in compositional data sets. Statistical Modelling, 15(2), 134158. https://doi.org/10.1177/1471082X14535524 
25. Martın-Fernandez, J. A., Barcelo-Vidal, C., \& Pawlowsky-Glahn, V. (2003). Dealing with Zeros and Missing Values in Compositional Data Sets Using Nonparametric Imputation. Mathematical Geology, 26.

26. Egozcue, J. J., Pawlowsky-Glahn, V., Mateu-Figueras, G., \& Barceló-Vidal, C. (2003). Isometric Logratio Transformations for Compositional Data Analysis. Mathematical Geology, 35(3), 279-300. https://doi.org/10.1023/A:1023818214614

27. Greenacre, M. (2019). Variable Selection in Compositional Data Analysis Using Pairwise Logratios. Mathematical Geosciences, 51(5), 649-682. https://doi.org/10.1007/s11004-018-9754-x

28. Lovell, D. R., Chua, X.-Y., \& McGrath, A. (2020). Counts: An outstanding challenge for log-ratio analysis of compositional data in the molecular biosciences. NAR Genomics and Bioinformatics, 2(2). https://doi.org/10.1093/nargab/lqaa040

29. Quinn, T. P., Erb, I., Gloor, G., Notredame, C., Richardson, M. F., \& Crowley, T. M. (2019). A field guide for the compositional analysis of any-omics data. GigaScience, 8(9), giz107. https://doi.org/10.1093/gigascience/giz107

30. Fernandes, A. D., Reid, J. N., Macklaim, J. M., McMurrough, T. A., Edgell, D. R., \& Gloor, G. B. (2014). Unifying the analysis of high-throughput sequencing datasets: Characterizing RNA-seq, 16S rRNA gene sequencing and selective growth experiments by compositional data analysis. Microbiome, 2 , 15. https://doi.org/10.1186/2049-2618-2-15

31. Mandal, S., Treuren, W. V., White, R. A., Eggesbø, M., Knight, R., \& Peddada, S. D. (2015). Analysis of composition of microbiomes: A novel method for studying microbial composition. Microbial Ecology in Health and Disease, 26(1), 27663. https://doi.org/10.3402/mehd.v26.27663

32. Lin, H., \& Peddada, S. D. (2020). Analysis of compositions of microbiomes with bias correction. Nature Communications, 11(1), 3514. https://doi.org/10.1038/s41467-020-17041-7

33. American Journal of Roentgenology. 2005;185: 299-309. 10.2214/ajr.185.2.01850299 
34. Layeghifard, M., Hwang, D. M., \& Guttman, D. S. (2017). Disentangling Interactions in the Microbiome: A Network Perspective. Trends in Microbiology, 25(3), 217-228. https://doi.org/10.1016/j.tim.2016.11.008

35. Anderson, M. J. (2017). Permutational Multivariate Analysis of Variance (PERMANOVA). In Wiley StatsRef: Statistics Reference Online (pp. 1-15). American Cancer Society. https://doi.org/10.1002/9781118445112.stat07841

36. Clarke, K. R. (1993). Non-parametric multivariate analyses of changes in community structure. Australian Journal of Ecology, 18(1), 117-143. https://doi.org/10.1111/j.1442-9993.1993.tb00438.x

37. Rizzo, M. L., \& Székely, G. J. (2016). Energy distance. Wiley Interdisciplinary Reviews: Computational Statistics, 8(1), 27-38. https://doi.org/10.1002/wics.1375

38. Wu, C., Chen, J., Kim, J., \& Pan, W. (2016). An adaptive association test for microbiome data. Genome Medicine, 8(1), 56. https://doi.org/10.1186/s13073-016-0302-3

39. Koh, H., \& Zhao, N. (2020). A powerful microbial group association test based on the higher criticism analysis for sparse microbial association signals. Microbiome, 8(1), 63. https://doi.org/10.1186/s40168-020-00834-9

40. Baumann, K. (2003). Cross-validation as the objective function for variable-selection techniques. TrAC Trends in Analytical Chemistry, 22(6), 395-406. https://doi.org/10.1016/S01659936(03)00607-1

41. Lindgren, F., Hansen, B., Karcher, W., Sjöström, M., \& Eriksson, L. (1996). Model validation by permutation tests: Applications to variable selection. Journal of Chemometrics, $10(5-6), 521-532$. https://doi.org/10.1002/(SICI)1099-128X(199609)10:5/6<521::AID-CEM448>3.0.CO;2-J

42. Wei, S., Lee, C., Wichers, L., \& Marron, J. S. (2016). Direction-Projection-Permutation for HighDimensional Hypothesis Tests. Journal of Computational and Graphical Statistics, 25(2), 549-569. https://doi.org/10.1080/10618600.2015.1027773 
43. Kursa, M. B., Jankowski, A., \& Rudnicki, W. R. (2010). Boruta - A System for Feature Selection. Fundamenta Informaticae, 101(4), 271-285. https://doi.org/10.3233/FI-2010-288

44. Tibshirani, R. (2011). Regression shrinkage and selection via the lasso: A retrospective.

45. KENT, J. T. (1983). Information gain and a general measure of correlation. Biometrika, 70(1), 163173. https://doi.org/10.1093/biomet/70.1.163

46. Granitto, P. M., Furlanello, C., Biasioli, F., \& Gasperi, F. (2006). Recursive feature elimination with random forest for PTR-MS analysis of agroindustrial products. Chemometrics and Intelligent Laboratory Systems, 83(2), 83-90. https://doi.org/10.1016/i.chemolab.2006.01.007

47. Anderson, M. J., \& Walsh, D. C. I. (2013). PERMANOVA, ANOSIM, and the Mantel test in the face of heterogeneous dispersions: What null hypothesis are you testing? Ecological Monographs, 83(4), 557-574. https://doi.org/10.1890/12-2010.1

48. Anderson, M. J. (2006). Distance-Based Tests for Homogeneity of Multivariate Dispersions. Biometrics, 62(1), 245-253.

49. Paulson, J. N., Williams, B. L., Hehnly, C., Mishra, N., Sinnar, S. A., Zhang, L., Ssentongo, P., MbabaziKabachelor, E., Wijetunge, D. S. S., von Bredow, B., Mulondo, R., Kiwanuka, J., Bajunirwe, F., Bazira, J., Bebell, L. M., Burgoine, K., Couto-Rodriguez, M., Ericson, J. E., Erickson, T., ... Schiff, S. J. (2020). Paenibacillus infection with frequent viral coinfection contributes to postinfectious hydrocephalus in Ugandan infants. Science Translational Medicine, 12(563), eaba0565. https://doi.org/10.1126/scitranslmed.aba0565

50. Oliveira, F. S., Brestelli, J., Cade, S., Zheng, J., lodice, J., Fischer, S., Aurrecoechea, C., Kissinger, J. C., Brunk, B. P., Stoeckert, C. J., Jr, Fernandes, G. R., Roos, D. S., \& Beiting, D. P. (2018). MicrobiomeDB: A systems biology platform for integrating, mining and analyzing microbiome experiments. Nucleic Acids Research, 46(D1), D684-D691. https://doi.org/10.1093/nar/gkx1027 
51. Paulson J et al. Study: PIH Uganda. MicrobiomeDB. 2020; release 18 (https://microbiomedb.org/mbio/app/record/dataset/DS 953b8ff2d4)

52. Bokulich, N. A., Chung, J., Battaglia, T., Henderson, N., Jay, M., Li, H., D. Lieber, A., Wu, F., PerezPerez, G. I., Chen, Y., Schweizer, W., Zheng, X., Contreras, M., Dominguez-Bello, M. G., \& Blaser, M. J. (2016). Antibiotics, birth mode, and diet shape microbiome maturation during early life. Science Translational Medicine, 8(343), 343ra82-343ra82. https://doi.org/10.1126/scitranslmed.aad7121

53. Gonzalez, A., Navas-Molina, J. A., Kosciolek, T., McDonald, D., Vázquez-Baeza, Y., Ackermann, G., DeReus, J., Janssen, S., Swafford, A. D., Orchanian, S. B., Sanders, J. G., Shorenstein, J., Holste, H., Petrus, S., Robbins-Pianka, A., Brislawn, C. J., Wang, M., Rideout, J. R., Bolyen, E., ... Knight, R. (2018). Qiita: Rapid, web-enabled microbiome meta-analysis. Nature Methods, 15(10), 796-798. https://doi.org/10.1038/s41592-018-0141-9

54. Proctor, L. M., Creasy, H. H., Fettweis, J. M., Lloyd-Price, J., Mahurkar, A., Zhou, W., Buck, G. A., Snyder, M. P., Strauss, J. F., Weinstock, G. M., White, O., Huttenhower, C., \& The Integrative HMP (iHMP) Research Network Consortium. (2019). The Integrative Human Microbiome Project. Nature, 569(7758), 641-648. https://doi.org/10.1038/s41586-019-1238-8

55. Lloyd-Price, J., Arze, C., Ananthakrishnan, A. N., Schirmer, M., Avila-Pacheco, J., Poon, T. W., Andrews, E., Ajami, N. J., Bonham, K. S., Brislawn, C. J., Casero, D., Courtney, H., Gonzalez, A., Graeber, T. G., Hall, A. B., Lake, K., Landers, C. J., Mallick, H., Plichta, D. R., ... Huttenhower, C. (2019). Multi-omics of the gut microbial ecosystem in inflammatory bowel diseases. Nature, $569(7758)$, 655-662. https://doi.org/10.1038/s41586-019-1237-9

56. Vatanen, T., Kostic, A. D., d’Hennezel, E., Siljander, H., Franzosa, E. A., Yassour, M., Kolde, R., Vlamakis, H., Arthur, T. D., Hämäläinen, A.-M., Peet, A., Tillmann, V., Uibo, R., Mokurov, S., Dorshakova, N., Ilonen, J., Virtanen, S. M., Szabo, S. J., Porter, J. A., ... Xavier, R. J. (2016). Variation in 
Microbiome LPS Immunogenicity Contributes to Autoimmunity in Humans. Cell, 165(4), 842-853.

$977 \quad$ https://doi.org/10.1016/i.cell.2016.04.007

978 57. Broad Institute. https://diabimmune.broadinstitute.org/diabimmune/. Accessed 23 May 2019.

979 58. Boogaart, K. G. van den, \& Tolosana-Delgado, R. (2013). Analyzing Compositional Data with R.

$980 \quad$ Springer-Verlag. https://doi.org/10.1007/978-3-642-36809-7

981 59. Palarea-Albaladejo, J., \& Martín-Fernández, J. A. (2015). zCompositions-R package for multivariate

982 imputation of left-censored data under a compositional approach. Chemometrics and Intelligent

983 Laboratory Systems, 143, 85-96. https://doi.org/10.1016/i.chemolab.2015.02.019

984 60. Fan, J., \& Lv, J. (2008). Sure independence screening for ultrahigh dimensional feature space. Journal

985 of the Royal Statistical Society: Series B (Statistical Methodology), 70(5), 849-911.

$986 \quad$ https://doi.org/10.1111/j.1467-9868.2008.00674.x

987 61. Csardi G, Nepusz T (2006). "The igraph software package for complex network research."

988 InterJournal, Complex Systems, 1695. https://igraph.org.

989 62. Rizzo, M. L., \& Székely, G. J. (2010). DISCO analysis: A nonparametric extension of analysis of

990 variance. The Annals of Applied Statistics, 4(2), 1034-1055. https://doi.org/10.1214/09-AOAS245

991 63. Ernst, M. D. (2004). Permutation Methods: A Basis for Exact Inference. Statistical Science, 19(4),

992 676-685. https://doi.org/10.1214/088342304000000396

993 64. Goodrich, J. K., Waters, J. L., Poole, A. C., Sutter, J. L., Koren, O., Blekhman, R., Beaumont, M.,

994 Van Treuren, W., Knight, R., Bell, J. T., Spector, T. D., Clark, A. G., \& Ley, R. E. (2014). Human Genetics

995 Shape the Gut Microbiome. Cell, 159(4), 789-799. https://doi.org/10.1016/j.cell.2014.09.053

996 65. Duvallet, Claire, Gibbons, Sean, Gurry, Thomas, Irizarry, Rafael, \& Alm, Eric. (2017). MicrobiomeHD:

997 the human gut microbiome in health and disease [Data set]. Zenodo.

$998 \quad$ http://doi.org/10.5281/zenodo.1146764 
66. Zeevi, D., Korem, T., Zmora, N., Israeli, D., Rothschild, D., Weinberger, A., Ben-Yacov, O., Lador, D., Avnit-Sagi, T., Lotan-Pompan, M., Suez, J., Mahdi, J. A., Matot, E., Malka, G., Kosower, N., Rein, M., Zilberman-Schapira, G., Dohnalová, L., Pevsner-Fischer, M., ... Segal, E. (2015). Personalized Nutrition by Prediction of Glycemic Responses. Cell, 163(5), 1079-1094. https://doi.org/10.1016/j.cell.2015.11.001

67. Pasolli E, Schiffer L, Manghi P, Renson A, Obenchain V, Truong D, Beghini F, Malik F, Ramos M, Dowd J, Huttenhower C, Morgan M, Segata N, Waldron L (2017). “Accessible, curated metagenomic data through ExperimentHub." Nat. Methods, 14(11), 1023-1024. ISSN 1548-7091, 1548-7105, doi: 10.1038/nmeth.4468.

68. Calgaro, M., Romualdi, C., Waldron, L., Risso, D., \& Vitulo, N. (2020). Assessment of statistical methods from single cell, bulk RNA-seq, and metagenomics applied to microbiome data. Genome Biology, 21(1), 191. https://doi.org/10.1186/s13059-020-02104-1

69. Susin, A., Wang, Y., Lê Cao, K.-A., \& Calle, M. L. (2020). Variable selection in microbiome compositional data analysis. NAR Genomics and Bioinformatics, 2(2). https://doi.org/10.1093/nargab/lqaa029

$1014 \quad$ Figures

1015 Fig. 1: Overview of the SelEnergyPerm framework for non-parametric group association testing in 1016 metagenomic data. a. Relative abundance/count data are transformed using all pairwise log ratios. 1017 These log ratios are subsequently scored (DCV) and used to efficiently select a subset that: (1) is 1018 independent via a maximum spanning tree, (2) maximizes the energy or association between groups via 1019 greedy optimization. The entire process is repeated using permutation testing to control false discovery 1020 and assess statistical significance. b. Detection/rejection of sparse associations hidden within high 1021 dimensional data via simultaneous feature selection and permutation testing. 
1022 Fig. 2: Comparison of SelEnergyPerm selected log ratio subsets characteristics with Boruta, Information

1023 Gain Filtering, LASSO, and RFE across five simulation scenarios with log ratio dimension $=$

$1024\left[\left(\begin{array}{c}50 \\ 2\end{array}\right),\left(\begin{array}{c}100 \\ 2\end{array}\right),\left(\begin{array}{c}150 \\ 2\end{array}\right),\left(\begin{array}{c}200 \\ 2\end{array}\right),\left(\begin{array}{c}250 \\ 2\end{array}\right)\right]$ for the balanced sampling design. Using 200 simulations for each

1025 scenario-dimension by method we assessed: (Top Row) the clustering coefficient of log ratio networks

1026 formed by selected subsets returned from each method, (Middle Row) the magnitude of the association

1027 as measured by the cF-statistic on selected subsets returned from each method, and (Bottom Row) the

1028 number of log ratios returned by each method. Points are the mean for each experimental condition

1029 and error bars indicate $95 \%$ confidence interval.

1030 Fig. 3: Comparison of the Matthews Correlation Coefficient measuring the ability of each method to

1031 properly detect/reject associations in data generated from synthetic distributions in both balanced and

1032 unbalanced sampling designs. For each scenario and log ratio feature space size $=\left[\left(\begin{array}{c}50 \\ 2\end{array}\right),\left(\begin{array}{c}150 \\ 2\end{array}\right),\left(\begin{array}{c}250 \\ 2\end{array}\right)\right]$

1033 test datasets were simulated to include data distribution that have either true between-group

1034 differences $(n=100)$ or no between-group difference $(n=100)$. Results from the PERMDISP2 procedure

1035 are displayed to indicate heterogeneity of variance between groups.

1036 Fig. 4: Comparison of the Matthews Correlation Coefficient measuring the ability of each method to

1037 properly detect/reject associations in data simulated from real $16 S$ and WGS data distributions in both

1038 balanced and unbalanced sampling designs. For each data type and scenario, datasets were generated

1039 to include data distribution that have either true between-group differences $(n=100)$ or no between-

1040 group difference $(n=100)$. Results from the PERMDISP2 (dashed line) procedure are displayed to indicate

1041 heterogeneity of variance between groups.

1042 Fig. 5: SelEnergyPerm case study examining the association between Ugandan infant's cerebrospinal

1043 fluid microbiomes and post-infectious hydrocephalus using $16 S$ data. a. SelEnergyPerm permutation test

1044 results displaying the null distribution of the cF statistic (Histogram, Density, and Points) and the 
1045

1046

1047

1048

1049

1050

1051

1052

1053

1054

1055

1056

1057

1058

1059

1060

1061

1062

1063

1064

1065

1066

1067

1068

empirical cF statistic (dashed red vertical line). b. Random forest (RF) importance weighted directed log ratio network (edges point from numerator to denominator) of the SelEnergyPerm selected signature (nodes $=$ taxa, node size $=$ weighted degree, edges $=$ log ratio, edge width $/$ color $=$ RF variable importance). c. ROC comparisons of disease status discrimination using RF. Models were trained with repeated $(r=50)$ 10-fold cross-validation using either the SelEnergyPerm Signature, all log ratios, or Paenibacillus alone. d. Principal component analysis using the SelEnergyPerm signature. e. (Left) Log ratio means of each log ratio included in the SelEnergyPerm signature. (Right) Loading weights of the first principal component. Significance codes $(*, * *, * * *, * * * *)$ indicate $\mathrm{BH}$ corrected $\mathrm{p}$-value $\leq(0.05$, $0.01,0.001,1 e-4,0)$ for between group Wilcoxon Rank Sum Test. For the log ratio means, positive values indicate numerator more abundant than the denominator and negative values indicate the denominator is more abundant numerator. Error bars indicate the $95 \% \mathrm{Cl}$ of the mean. Notably, error bars that do not span 0 indicate numerator/denominator is on average more abundant than the opposite.

Fig. 6: SelEnergyPerm case study examining the association between delivery mode and the gut microbiome composition of infants over the first three months of life using $16 \mathrm{~S}$ data. a. SelEnergyPerm permutation test (permutations $=1000$ ) results displaying the null distribution of the test statistic (violin and grey points) and the empirical test statistic (red if significant, black otherwise) with BenjaminiHochberg corrected $p$ values. Test statistics values were z-score scaled (by Collection Month) for ease of visualization. b. AUC comparisons of delivery mode discrimination using PLS-DA. Models were trained with repeated $(r=20) 5$-fold stratified (delivery mode and host) cross-validation using either the SelEnergyPerm signature or all log ratios. Points represent the mean AUC and error bars indicate the 95\% Cl. c. Relative taxa strength by family measuring the importance of each taxon for discriminating between delivery modes across each collection time point. Relative strength was computed using the top 5 nodes derived from the PLS-DA variable importance weighted log ratio networks across each collection time. d. Directed (edges point from numerator to denominator) network of the 
1070 (nodes $=$ taxa, node size $=$ mean strength, edge $=$ log ratio, edge width $=$ log ratio mean, red edges $=$ 1071 negative log ratio mean (incoming node more abundant), blue edges = positive log ratio mean (outgoing 1072 node more abundant)).

1073 Fig. 7: SelEnergyPerm case study examining the association between abnormal fecal calprotectin levels 1074 and the gut microbiome composition in nonIBD and IBD individuals using WGS data. a. SelEnergyPerm 1075 permutation test results displaying the null distribution of the cF statistic (Histogram, Density, and 1076 Points) and the empirical cF statistic (dashed red vertical line). b. AUC comparisons of fecal calprotectin 1077 level (Abnormal/Normal) discrimination using PLS-DA with 2 components. Models were trained with 1078 repeated $(r=20)$ 10-fold cross-validation using either the SelEnergyPerm signature or all log ratios. 1079 Points represent the mean AUC and error bars indicate the $95 \% \mathrm{Cl}$. c. Directed network (edges point 1080 from numerator to denominator) of the SelEnergyPerm-selected log-ratio signature (nodes = taxa, node 1081 size $=$ DCV strength, edges $=$ log ratio, edge width/color $=$ PLS-DA Variable Importance). The top 5 taxa 1082 names by strength (PLS-DA Variable Importance) are displayed. c. PLS-DA latent space projection plot 1083 extracted from final PLS-DA model fit using the full dataset with the SelEnergyPerm signature. Points 1084 represent non-IBD or IBD samples. d. Log ratio means of each log ratio included in the SelEnergyPerm 1085 signature demonstrating shared and distinct log ratio markers across diagnosis. Positive values indicate 1086 numerator more abundant than the denominator and negative values indicate the denominator is more 1087 abundant numerator. Significance codes $(\mathrm{ns}, *, * *, * * *, * * * *)$ indicate $\mathrm{BH}$ corrected $\mathrm{p}$-value $\leq$ (Not 1088 Significant, 0.05, 0.01, 0.001, 1e-4, 0) for between group Wilcoxon Rank Sum Test. Error bars indicate 1089 the $95 \% \mathrm{Cl}$ of the mean. Notably, error bars that do not span 0 indicate numerator/denominator is on 1090 average more abundant than the opposite.

1091 Fig. 8: SelEnergyPerm case study examining the association between the gut microbiomes of infants in 1092 early life and the development of food allergy later in life. a. SelEnergyPerm permutation test 
1093 (permutations $=1000)$ results displaying the null distribution of the test statistic (violin and grey points) 1094 and the empirical test statistic (red if significant, black otherwise) with Benjamini-Hochberg corrected $p$ 1095 values. Test statistics values were $z$-score scaled by collection period to improve visualization. b. AUC 1096 comparisons of future food allergy development discrimination using PLS-DA. Models were trained with 1097 repeated $(r=20)$ 10-fold stratified (host and food allergy development) cross-validation using either the 1098 SelEnergyPerm signature or all log ratios. Points represent the mean AUC and error bars indicate the $109995 \% \mathrm{Cl}$. c. Relative taxa strength by family measuring the importance of each taxon for discriminating 1100 between food allergy statuses later in life across each collection month. Relative strength was computed 1101 using the top 5 nodes derived from the PLS-DA variable importance weighted log ratio networks across 1102 each collection month. d. Directed (edges point out from numerator to denominator) network of the 1103 SelEnergyPerm-derived signature by collection period and food allergy development weighted by the 1104 absolute log ratio means (nodes $=$ taxa, node size $=$ mean strength, edge $=$ log ratio, edge width $=\log$ 1105 ratio mean, red edges $=$ negative log ratio mean (incoming node more abundant), blue edges $=$ positive 1106 log ratio mean (outgoing node more abundant)).

1107 Additional File 1: Figure S1: Feature selection computational time comparisons for balanced and 1108 unbalanced sampling designs between SelEnergyPerm, LASSO, RFE, RF, Information Gain, and Boruta 1109 across each scenario and dimension. Points are the mean for each experimental condition.

1110 Additional File 2: Figure S2: Comparison of SelEnergyPerm selected log ratio subsets characteristics 1111 with Boruta, Information Gain Filtering, LASSO, and RFE across five simulation scenarios with log ratio 1112 dimension $=\left[\left(\begin{array}{c}50 \\ 2\end{array}\right),\left(\begin{array}{c}100 \\ 2\end{array}\right),\left(\begin{array}{c}150 \\ 2\end{array}\right),\left(\begin{array}{c}200 \\ 2\end{array}\right),\left(\begin{array}{c}250 \\ 2\end{array}\right)\right]$ for the unbalanced sampling design. Using 200 1113 simulations for each scenario-dimension by method we assessed: (Top Row) the clustering coefficient of 1114 log ratio networks formed by selected subsets returned from each method, (Middle Row) the magnitude 1115 of the association as measured by the cF-statistic on selected subsets returned from each method, and 
1116 (Bottom Row) the number of log ratios returned by each method. Points are the mean for each

1117 experimental condition and error bars indicate 95\% confidence interval.

1118 Additional File 3: Figure S3: Overall mean performance comparison for data generated from synthetic

1119 distributions aggregated across all scenarios and dimensions using MCC, Sensitivity, Specify, Positive

1120 predictive value (PPV), Negative predictive value (NPV), Youden Index, and False Positive Rate (FPR)

1121 metric. Error bars indicate standard error.

1122 Additional File 4: Figure S4: Overall mean performance comparison for data generated from $16 \mathrm{~S}$ and

1123 WGS synthetic data aggregated across all scenarios and effect levels using MCC, Sensitivity, Specify,

1124 Positive predictive value (PPV), Negative predictive value (NPV), Youden Index, and False Positive Rate

1125 (FPR) metric. Error bars indicate standard error.

1126

1127

1128

1129 


\section{Figures}

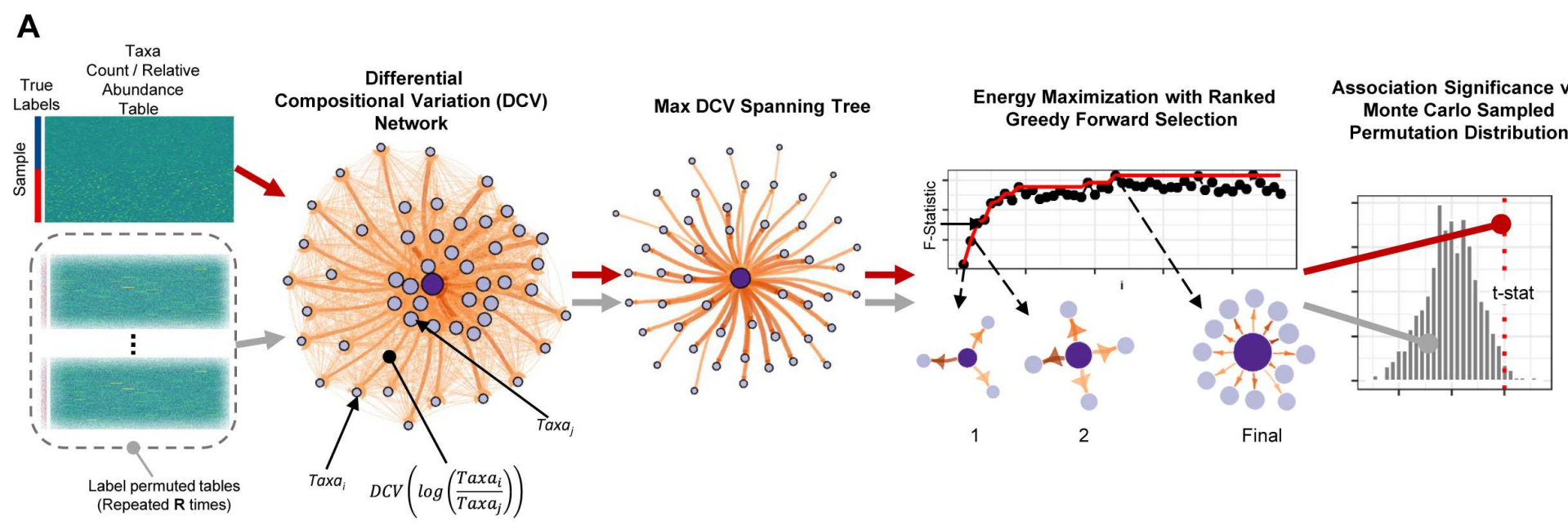

B
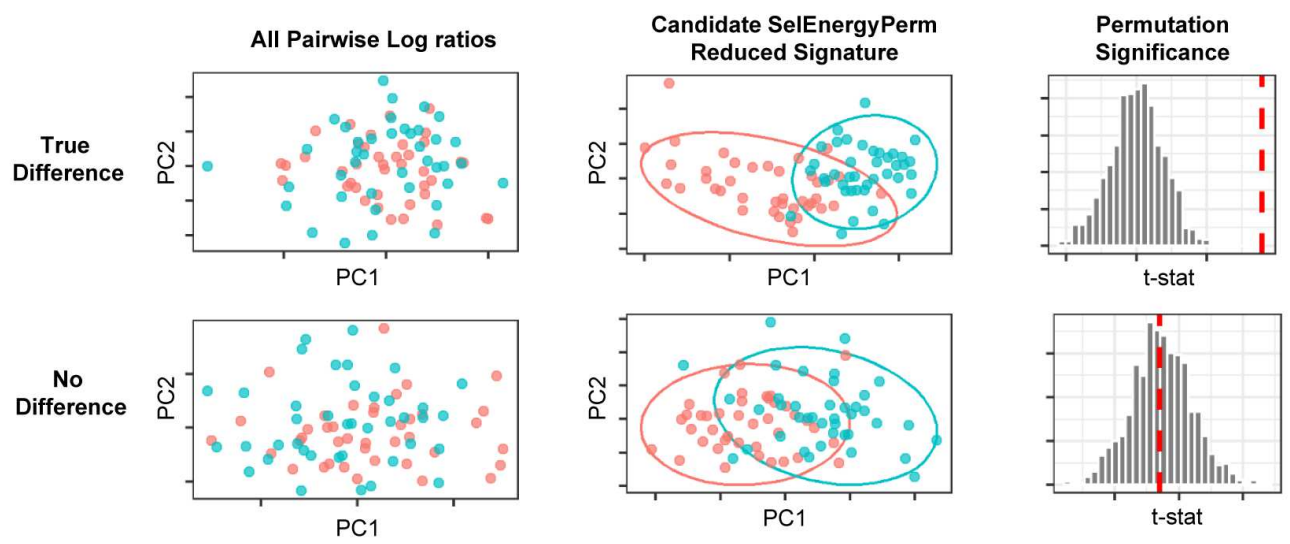

\section{Figure 1}

Overview of the SelEnergyPerm framework for non-parametric group association testing in metagenomic data. a. Relative abundance/count data are transformed using all pairwise log ratios. These log ratios are subsequently scored (DCV) and used to efficiently select a subset that: (1) is independent via a maximum spanning tree, (2) maximizes the energy or association between groups via greedy optimization. The entire process is repeated using permutation testing to control false discovery and assess statistical significance. b. Detection/rejection of sparse associations hidden within high dimensional data via simultaneous feature selection and permutation testing. 


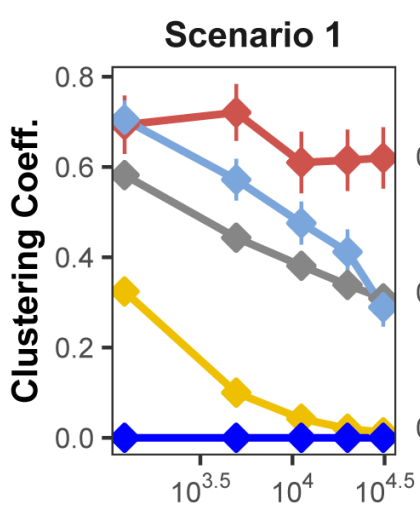

Boruta $\gamma$ InformationGain $\psi$ LASSO
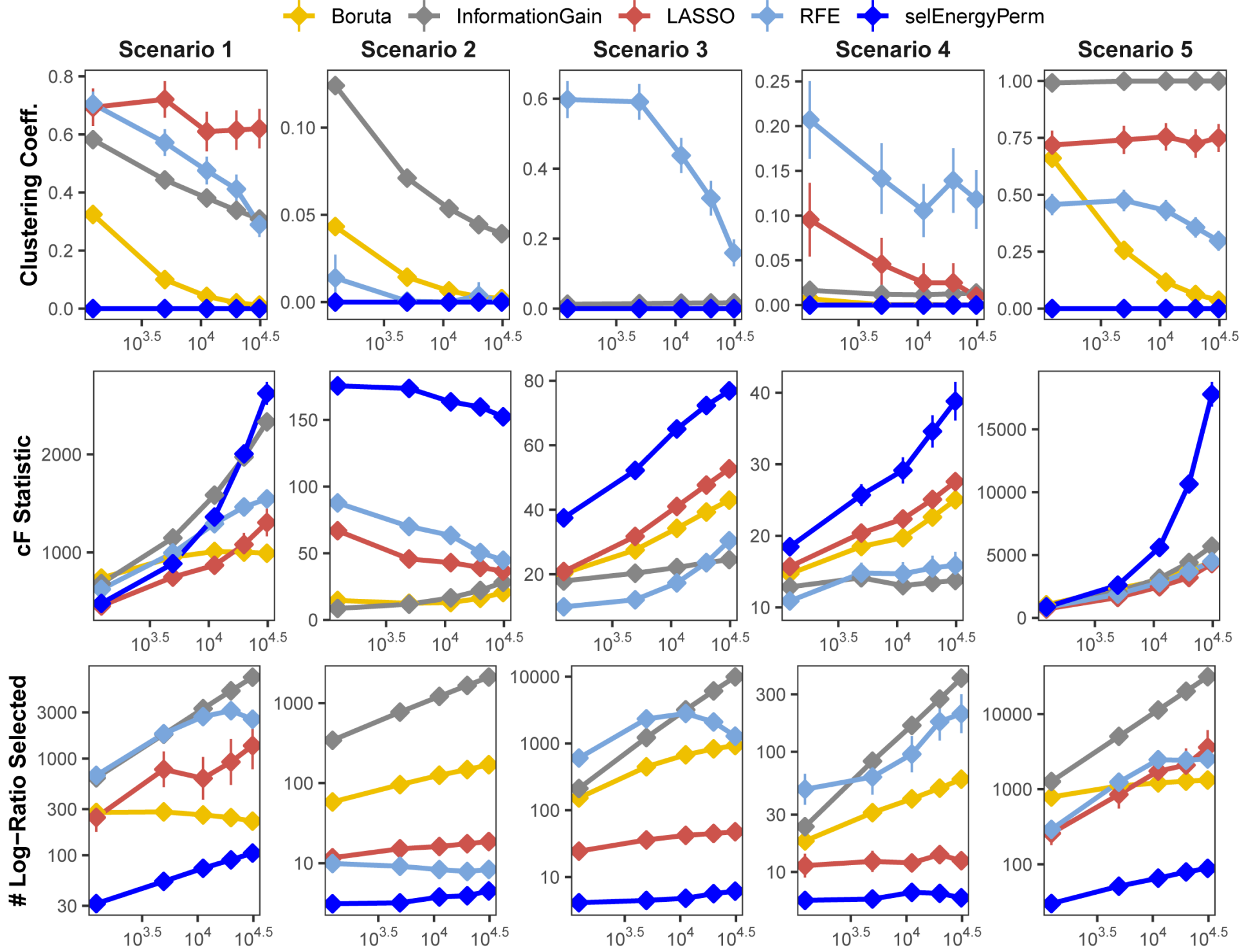

Log-ratio Dimensions

Figure 2

Due to technical limitations, the figure 2 caption is only available in the manuscript file. 


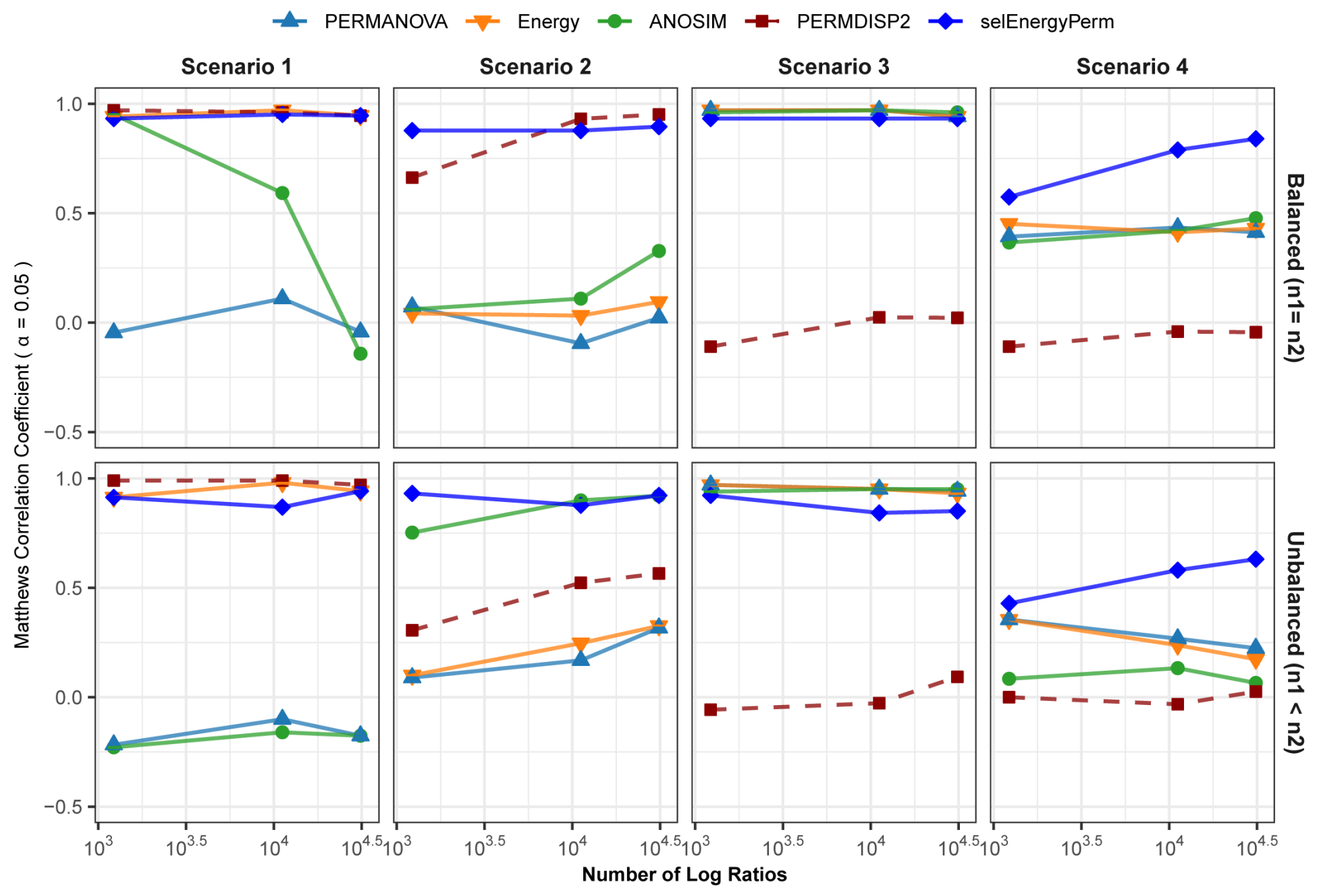

Figure 3

Due to technical limitations, the figure 3 caption is only available in the manuscript file. 


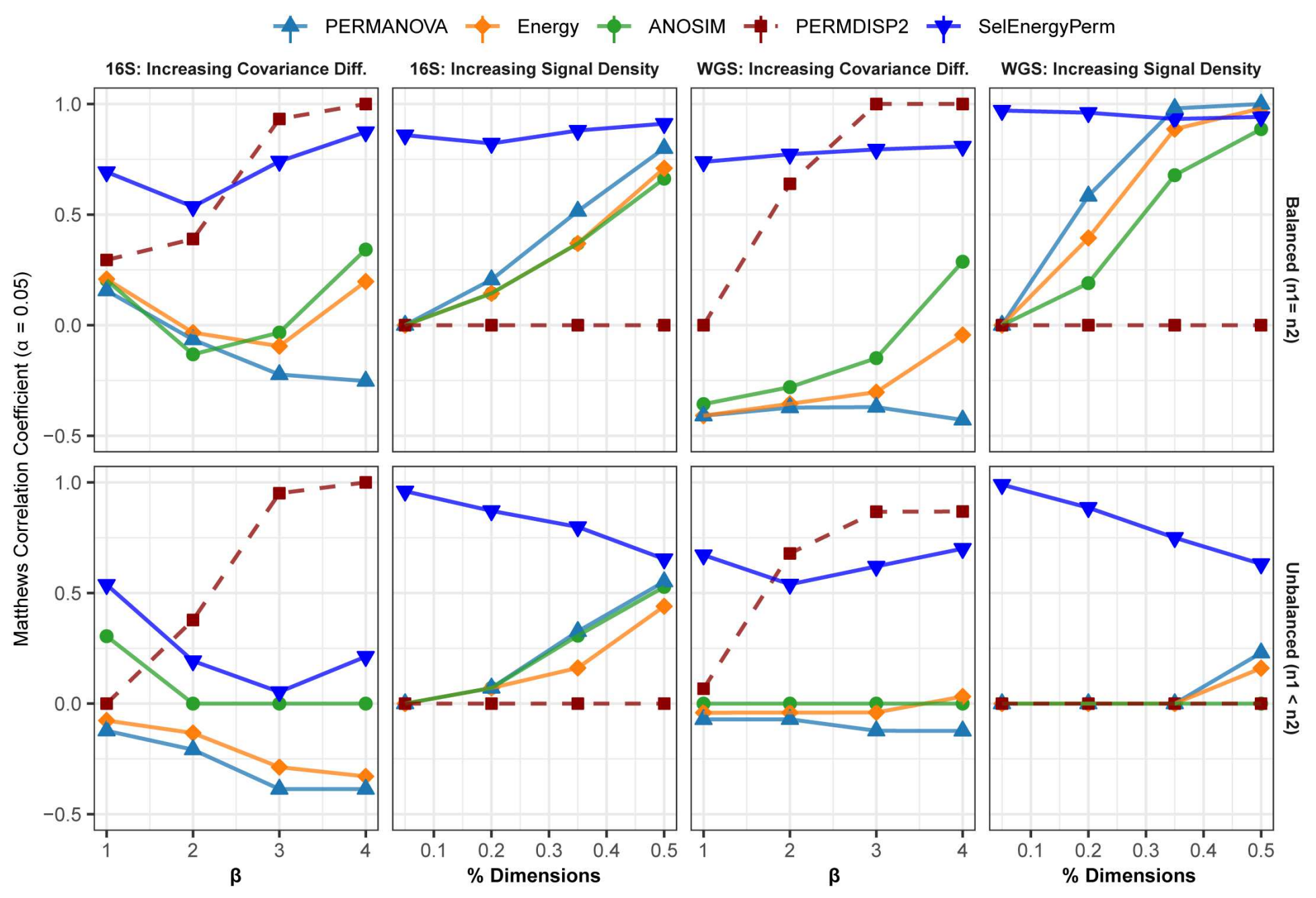

Figure 4

Comparison of the Matthews Correlation Coefficient measuring the ability of each method to properly detect/reject associations in data simulated from real $16 \mathrm{~S}$ and WGS data distributions in both balanced and unbalanced sampling designs. For each data type and scenario, datasets were generated to include data distribution that have either true between-group differences $(n=100)$ or no between- group difference $(n=100)$. Results from the PERMDISP2 (dashed line) procedure are displayed to indicate heterogeneity of variance between groups. 
A

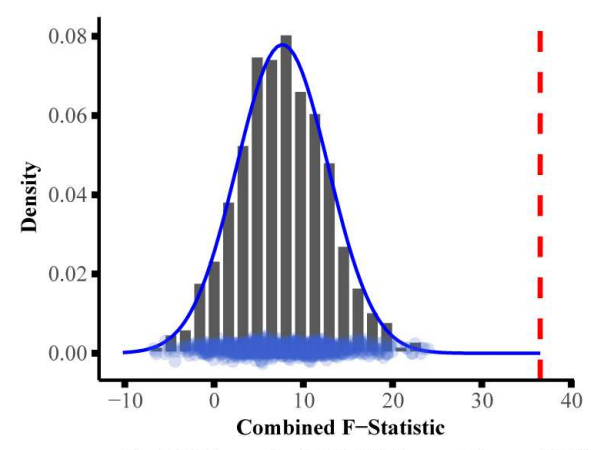

$\mathrm{cF}=36.521, \mathrm{pval}=0.001998$ (permutations $=1000$

E

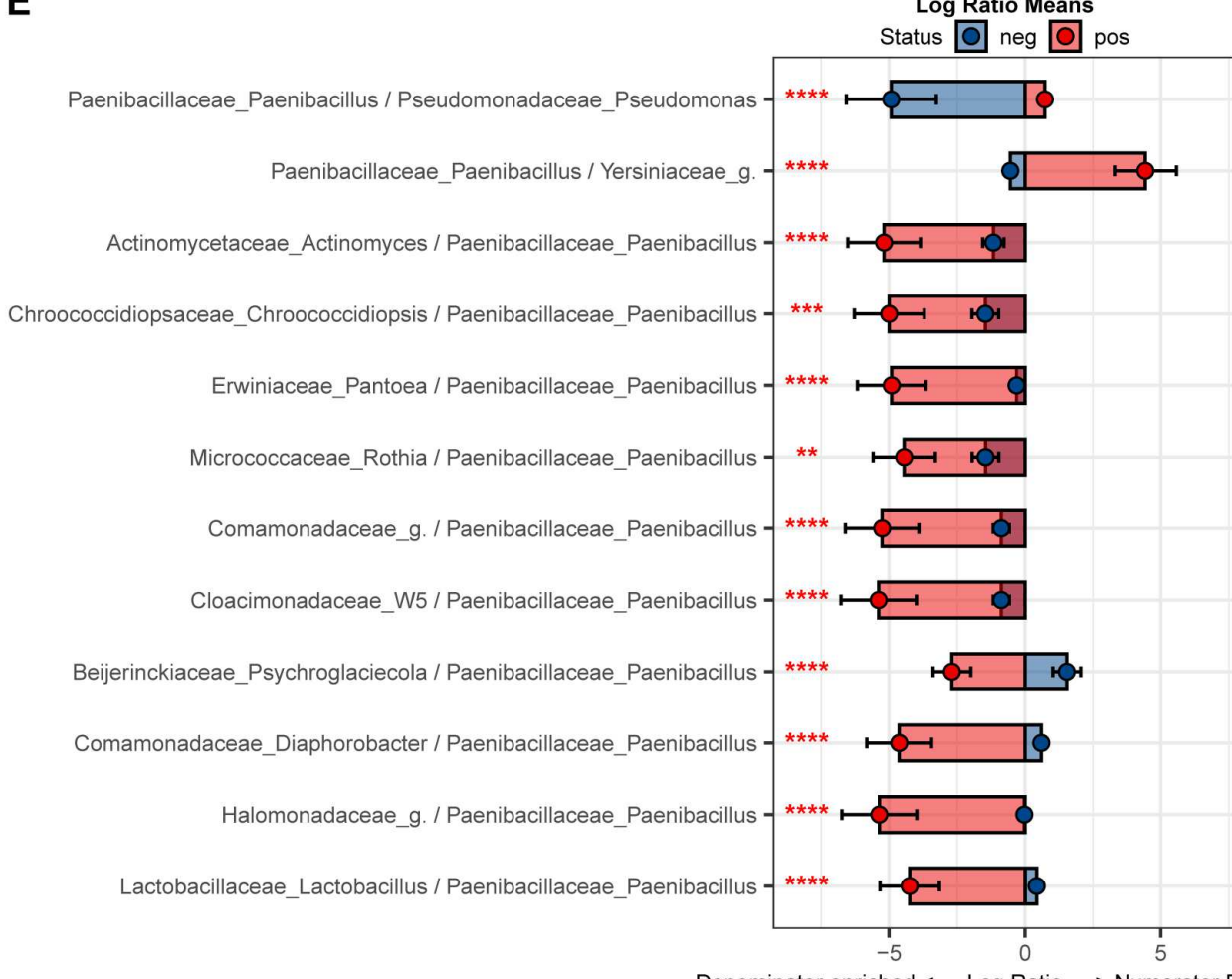

B

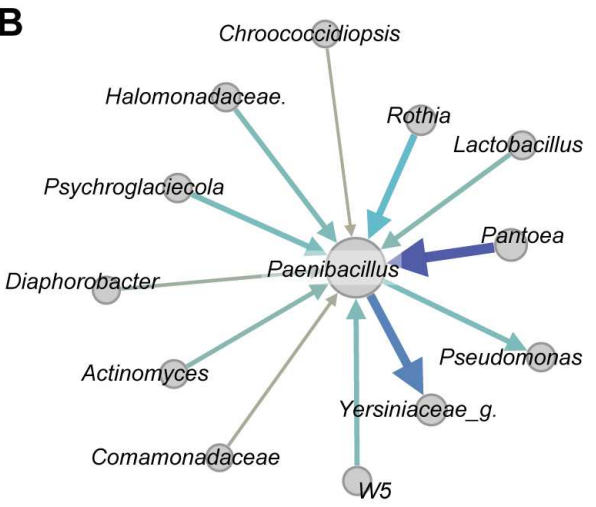

C - all logratios - Paenibacillus alone - signature

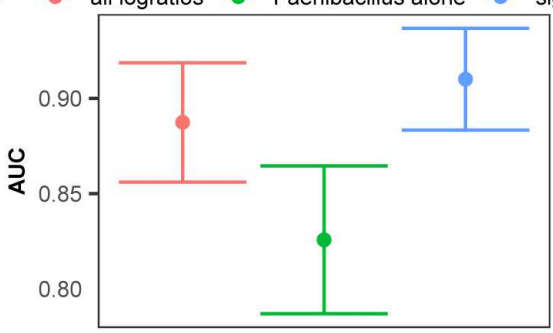

D
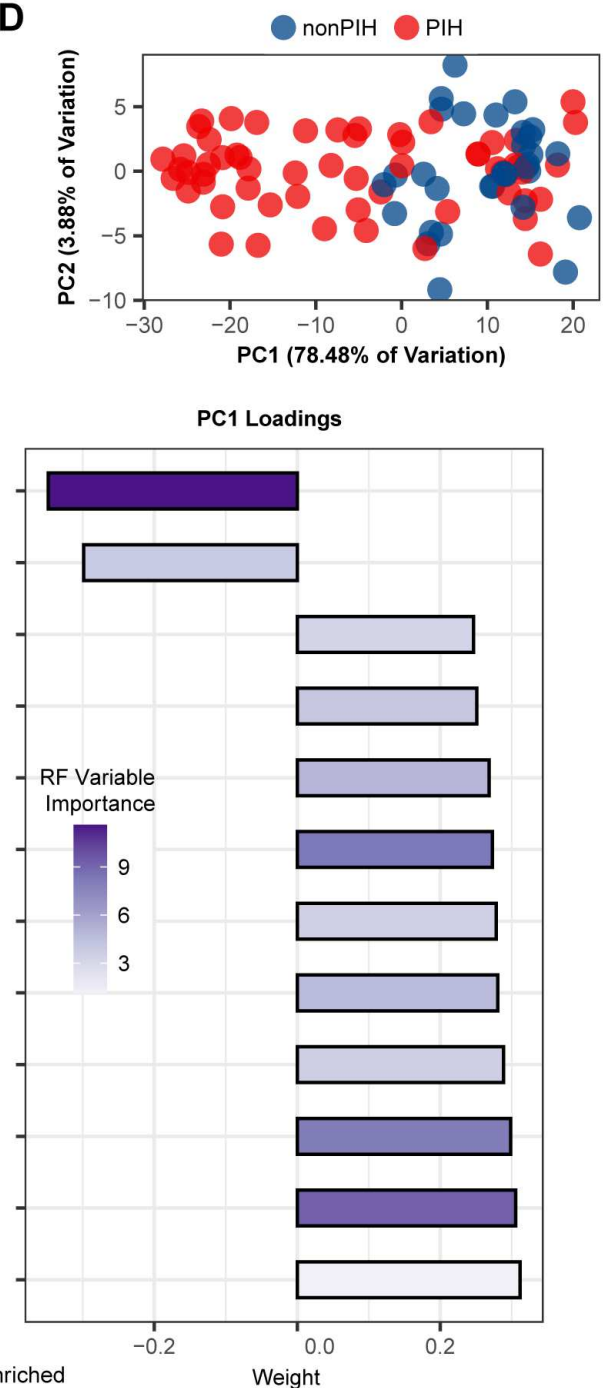

\section{Figure 5}

SelEnergyPerm case study examining the association between Ugandan infant's cerebrospinal fluid microbiomes and post-infectious hydrocephalus using 16S data. a. SelEnergyPerm permutation test results displaying the null distribution of the cF statistic (Histogram, Density, and Points) and the 49 empirical cF statistic (dashed red vertical line). b. Random forest (RF) importance weighted directed log ratio network (edges point from numerator to denominator) of the SelEnergyPerm selected signature (nodes $=$ taxa, node size $=$ weighted degree edges $=\log$ ratio, edge width/color $=$ RF variable importance) . c. ROC comparisons of disease status discrimination using RF. Models were trained with repeated $(r=50)$ 10 -fold cross-validation using either the SelEnergyPerm Signature, all log ratios, or Paenibacillus alone. $d$. 
Principal component analysis using the SelEnergyPerm signature. e. (Left) Log ratio means of each log ratio included in the SelEnergyPerm signature. (Right) Loading weights of the first principal component. Significance codes $(*, * \star, * \star *, * \star * *)$ indicate BH corrected p-value $<(0.05,10520.01,0.001,1 \mathrm{e}-4,0)$ for between group Wilcoxon Rank Sum Test. For the log ratio means, positive values indicate numerator more abundant than the denominator and negative values indicate the denominator is more abundant numerator. Error bars indicate the $95 \% \mathrm{Cl}$ of the mean. Notably, error bars that do not span 0 indicate numerator/denominator is on average more abundant than the opposite.

A

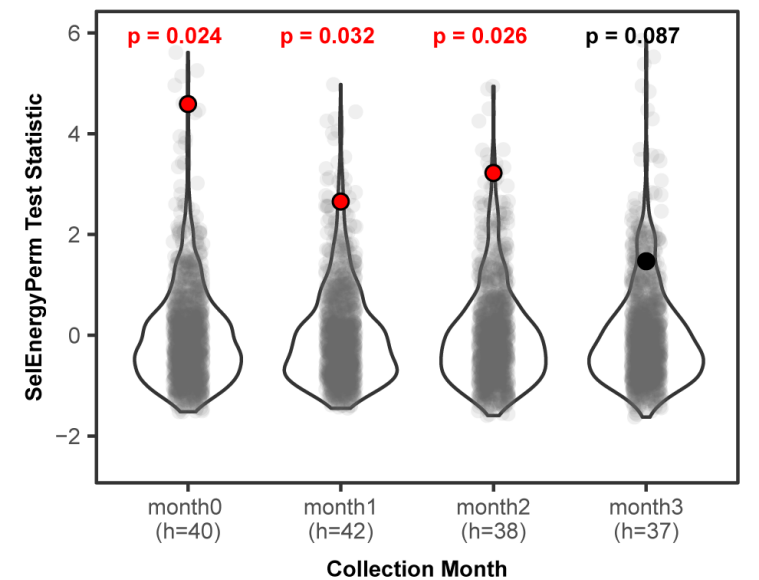

D
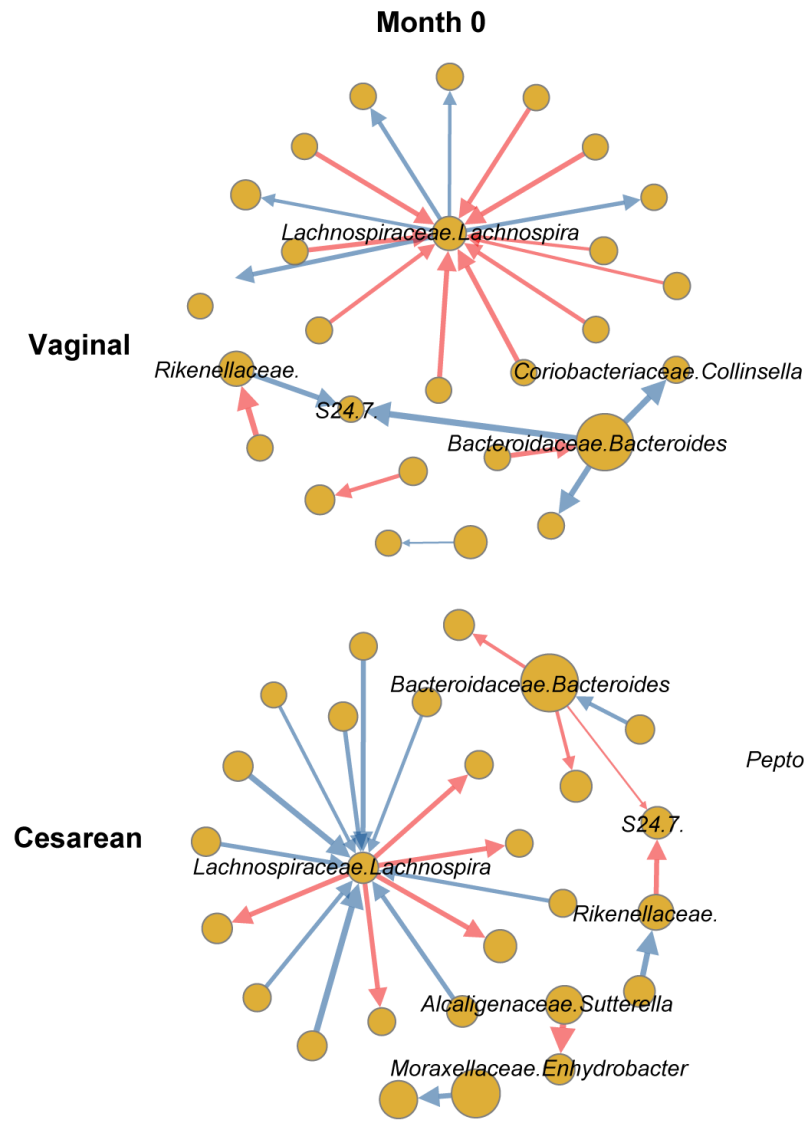

B

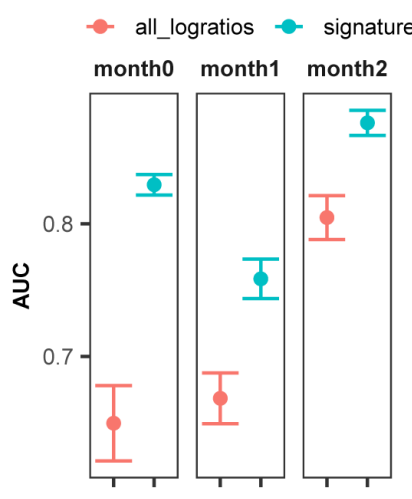

C

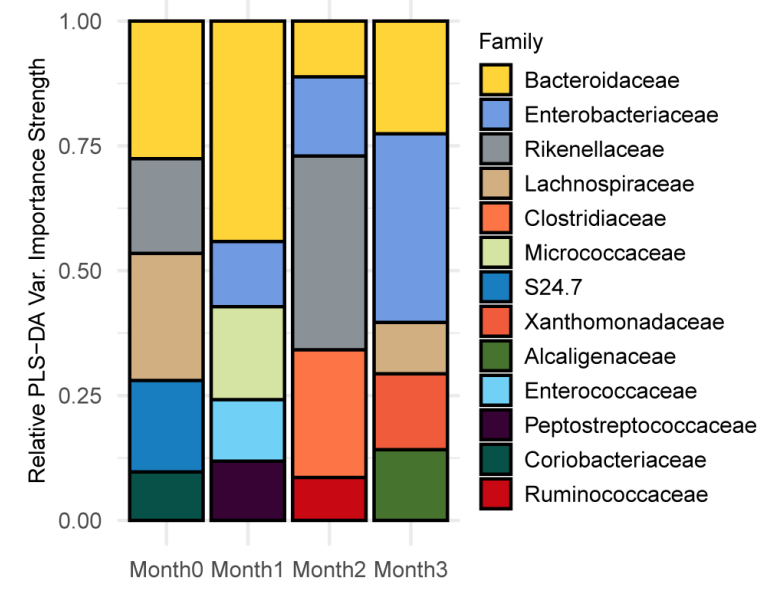

Month 1
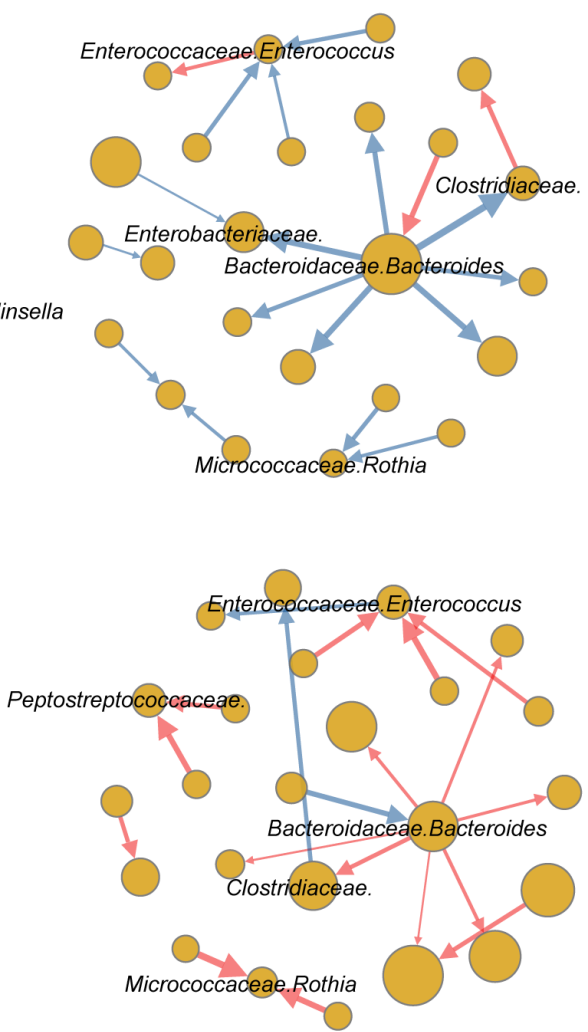

Month 2

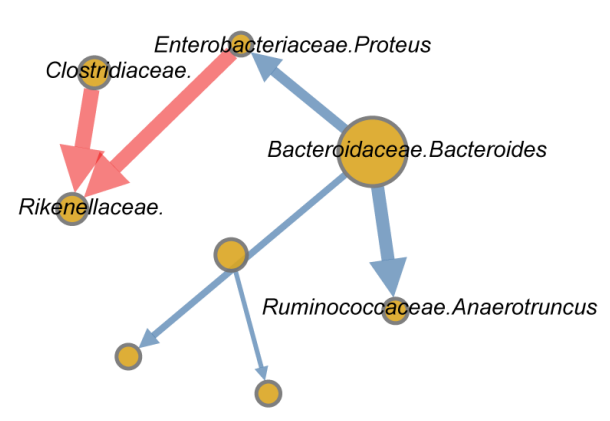

Figure 6 
SelEnergyPerm case study examining the association between delivery mode and the gut microbiome composition of infants over the first three months of life using 16S data. a. SelEnergyPerm permutation test (permutations $=1000$ ) results displaying the null distribution of the test statistic (violin and grey points) and the empirical test statistic (red if significant, black otherwise) with Benjamini-Hochberg corrected $p$ values. Test statistics values were z-score scaled (by Collection Month) for ease of visualization. b. AUC comparisons of delivery mode discrimination using PLS-DA. Models were trained with repeated $(r=20) 5$-fold stratified (delivery mode and host) cross-validation using either the SelEnergyPerm signature or all log ratios. Points represent the mean AUC and error bars indicate the $95 \%$ Cl. c. Relative taxa strength by family measuring the importance of each taxon for discriminating between delivery modes across each collection time point. Relative strength was computed using the top 5 nodes derived from the PLS-DA variable importance weighted log ratio networks across each collection time. d. Directed (edges point from numerator to denominator) network of the 50 SelEnergyPerm-derived signature by month and delivery mode weighted by the absolute log ratio means (nodes = taxa, node size $=$ mean strength, edge $=\log$ ratio, edge width $=\log$ ratio mean, red edges = negative log ratio mean (incoming node more abundant), blue edges = positive log ratio mean (outgoing node more abundant)). 
A

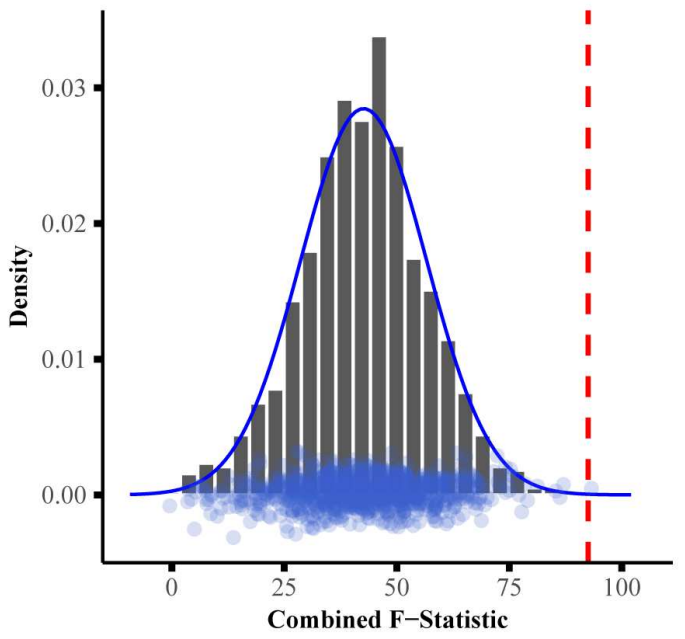

$\mathrm{cF}=92.507, \mathrm{pval}=0.000999($ permutations $=1000)$
B

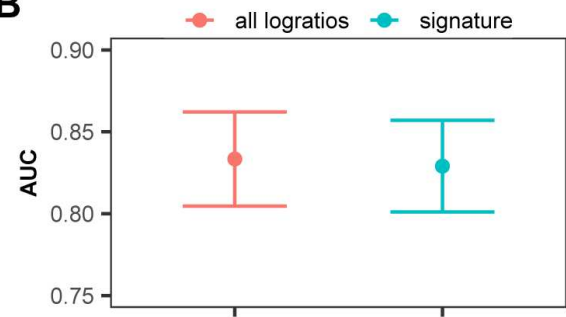

C

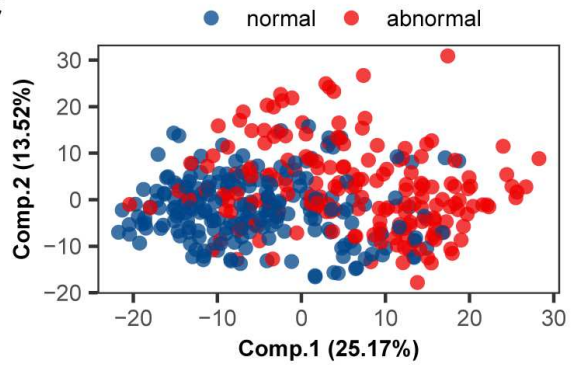

D

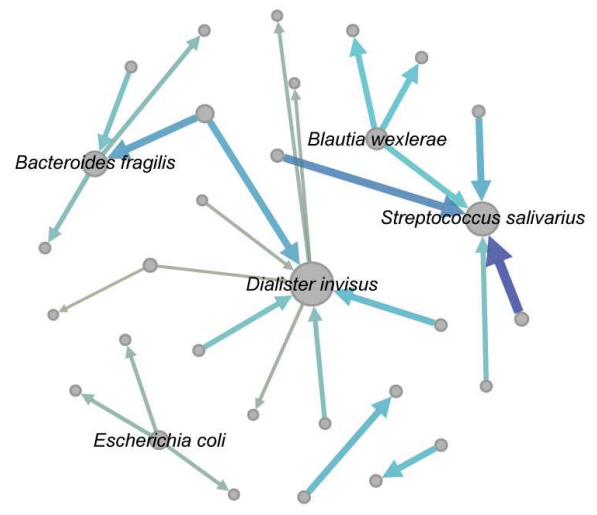

$\mathbf{E}$

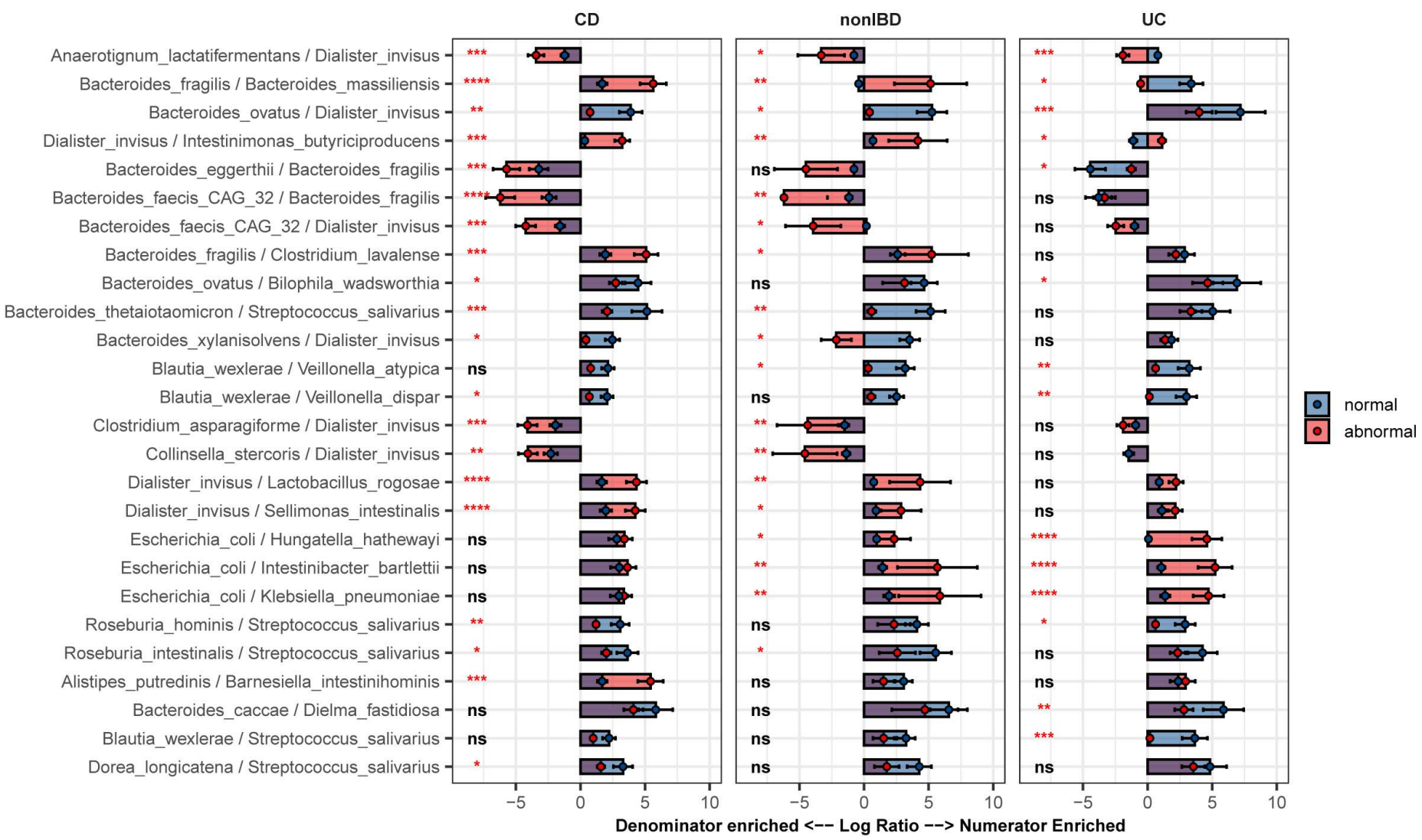

Figure 7

SelEnergyPerm case study examining the association between abnormal fecal calprotectin levels and the gut microbiome composition in nonIBD and IBD individuals using WGS data. a. SelEnergyPerm permutation test results displaying the null distribution of the cF statistic (Histogram, Density, and Points) and the empirical cF statistic (dashed red vertical line). b. AUC comparisons of fecal calprotectin level (Abnormal/Normal) discrimination using PLS-DA with 2 components. Models were trained with repeated $(r=20) 10$-fold cross-validation using either the SelEnergyPerm signature or all log ratios. Points represent the mean AUC and error bars indicate the $95 \% \mathrm{Cl}$. c. Directed network (edges point from 
numerator to denominator) of the SelEnergyPerm-selected log-ratio signature (nodes $=$ taxa, node size $=$ DCV strength, edges $=$ log ratio, edge width/color $=$ PLS-DA Variable Importance). The top 5 taxa names by strength (PLS-DA Variable Importance) are displayed. c. PLS-DA latent space projection plot extracted from final PLS-DA model fit using the full dataset with the SelEnergyPerm signature. Points represent non-IBD or IBD samples. d. Log ratio means of each log ratio included in the SelEnergyPerm signature demonstrating shared and distinct log ratio markers across diagnosis. Positive values indicate numerator more abundant than the denominator and negative values indicate the denominator is more abundant

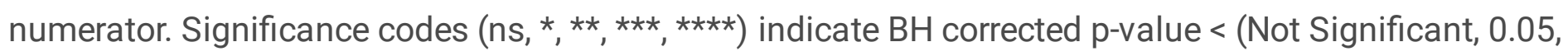
$0.01,0.001,1 \mathrm{e}-4,0)$ for between group Wilcoxon Rank Sum Test. Error bars indicate the $95 \% \mathrm{Cl}$ of the mean. Notably, error bars that do not span 0 indicate numerator/denominator is on average more abundant than the opposite. 
A

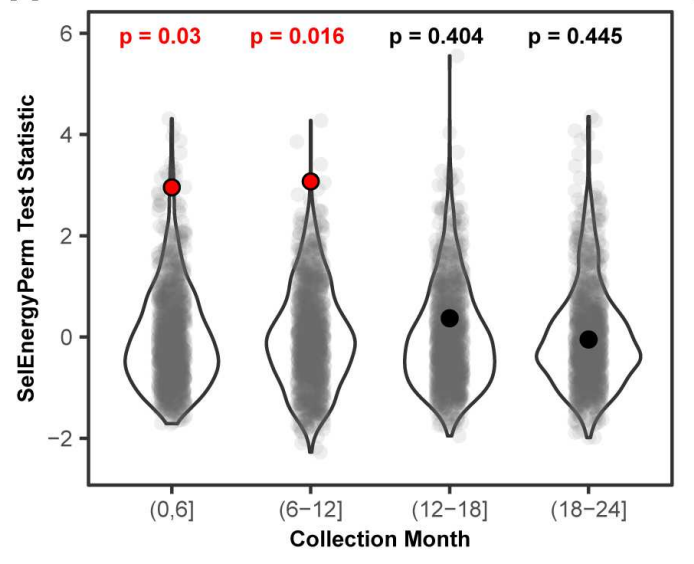

D

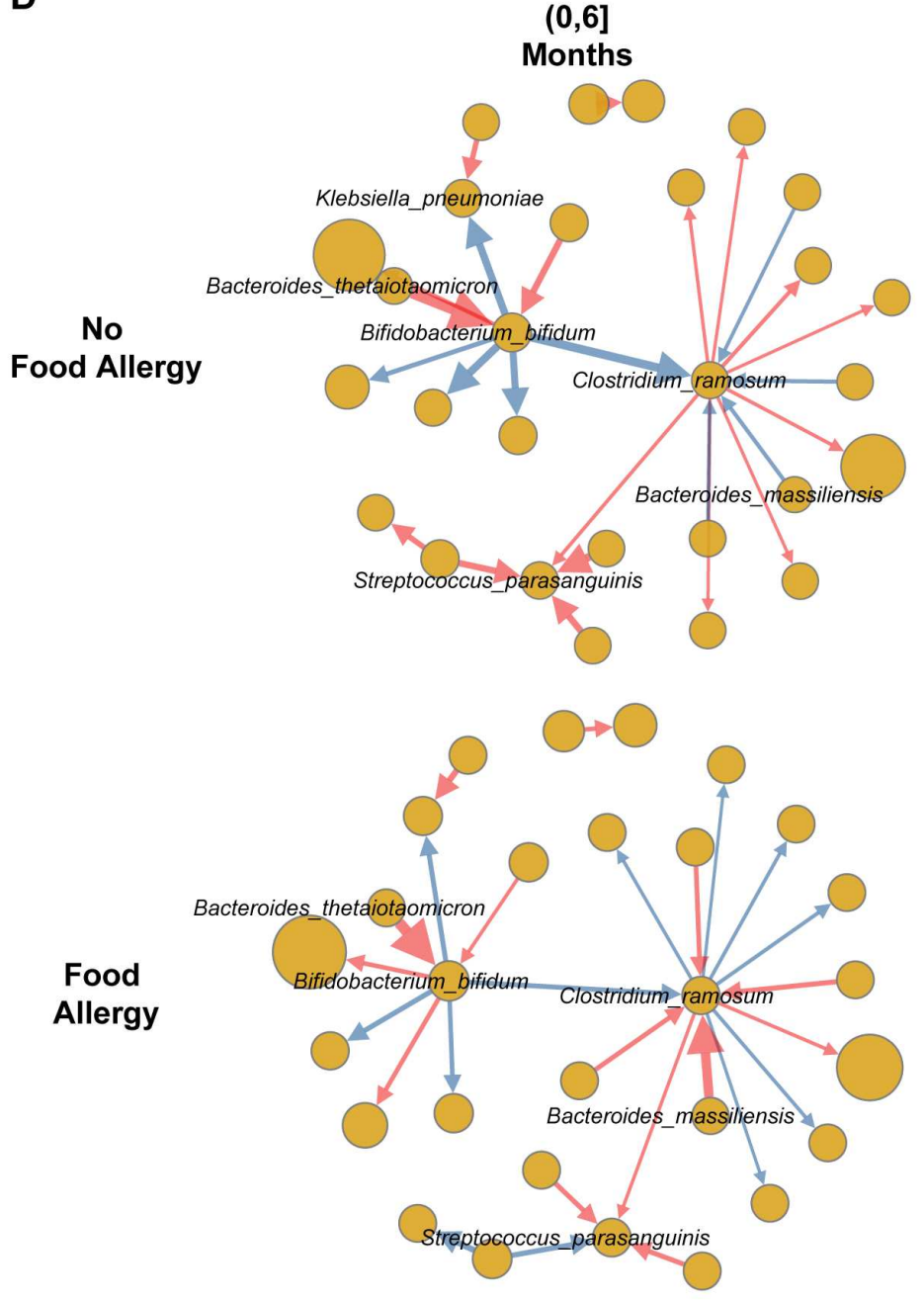

B $\rightarrow$ All Logratios - Signature $\quad$ C
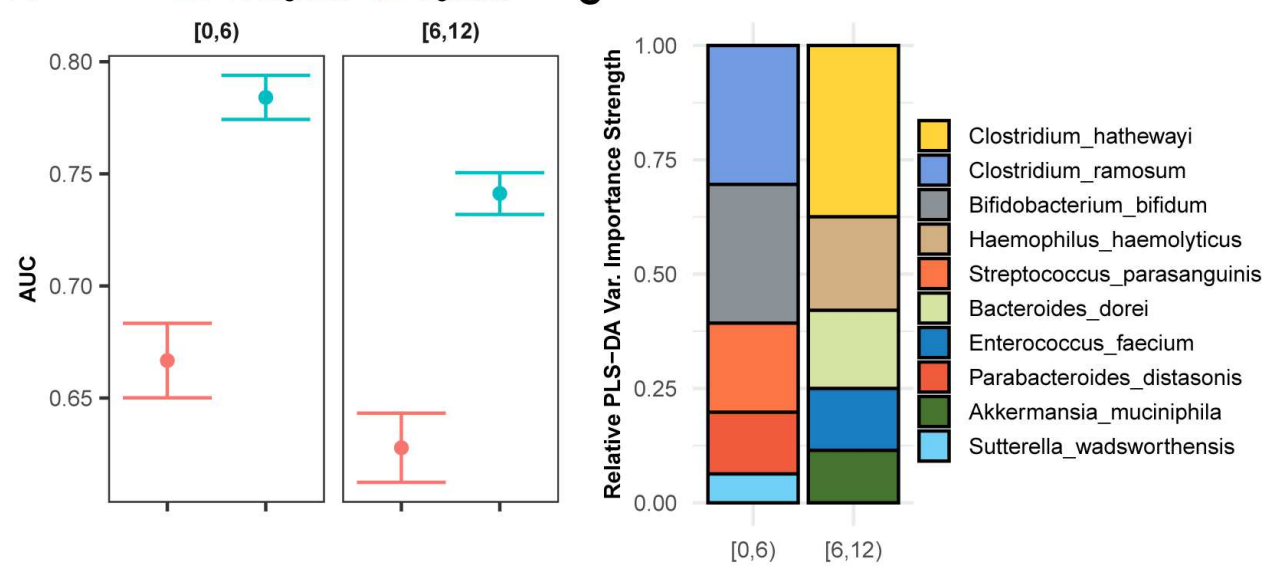

$(6,12]$ Months
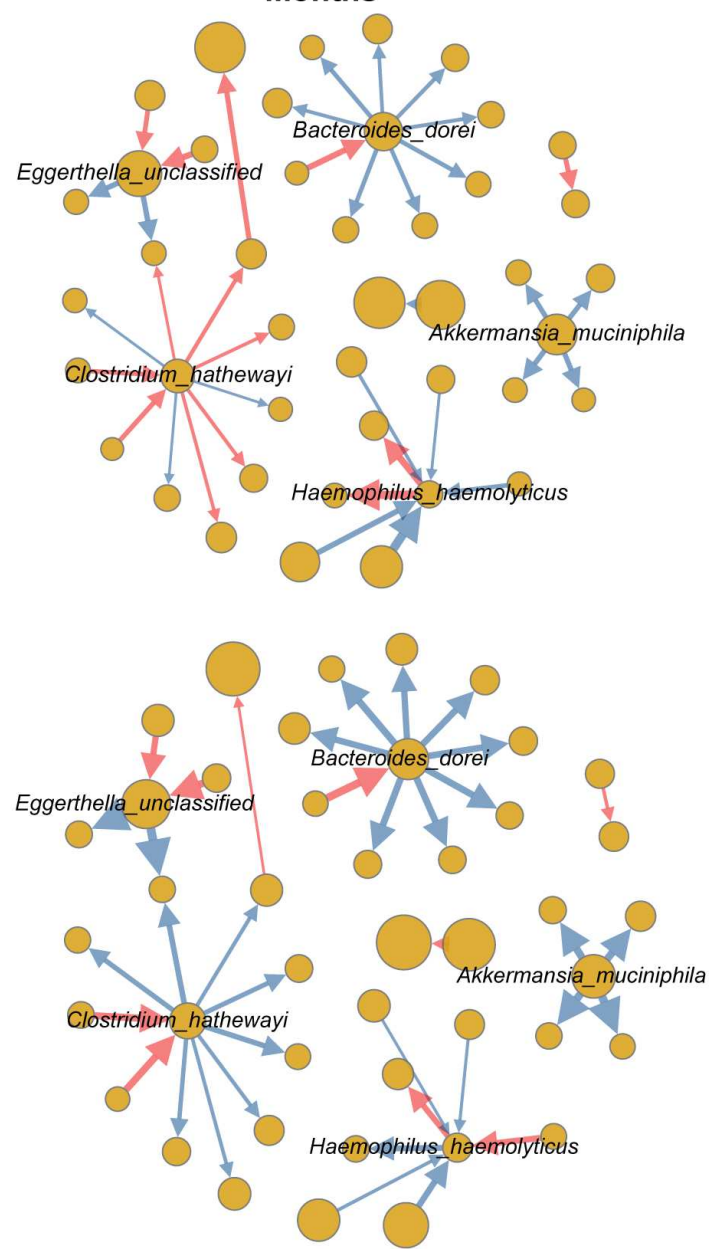

\section{Figure 8}

SelEnergyPerm case study examining the association between the gut microbiomes of infants in early life and the development of food allergy later in life. a. SelEnergyPerm permutation test 5 (permutations = 1000) results displaying the null distribution of the test statistic (violin and grey points) and the empirical test statistic (red if significant, black otherwise) with Benjamini-Hochberg corrected $p$ values. Test statistics values were z-score scaled by collection period to improve visualization. b. AUC comparisons of future food allergy development discrimination using PLS-DA. Models were trained with repeated $(r=20)$ 
10-fold stratified (host and food allergy development) cross-validation using either the SelEnergyPerm signature or all log ratios. Points represent the mean AUC and error bars indicate the $95 \% \mathrm{Cl}$. c. Relative taxa strength by family measuring the importance of each taxon for discriminating between food allergy statuses later in life across each collection month. Relative strength was computed using the top 5 nodes derived from the PLS-DA variable importance weighted log ratio networks across each collection month. d. Directed (edges point out from numerator to denominator) network of the SelEnergyPerm-derived signature by collection period and food allergy development weighted by theabsolute log ratio means (nodes $=$ taxa, node size $=$ mean strength, edge $=\log$ ratio, edge width $=\log$ ratio mean, red edges $=$ negative log ratio mean (incoming node more abundant), blue edges = positive log ratio mean (outgoing node more abundant)).

\section{Supplementary Files}

This is a list of supplementary files associated with this preprint. Click to download.

- AdditionalFile1.tif

- AdditionalFile2.tif

- AdditionalFile3.tif

- AdditionalFile4.tif 